J. DIFFERENTIAL GEOMETRY

71 (2005) 1-22

\title{
RIGIDITY OF SINGULAR SCHUBERT VARIETIES IN $\operatorname{Gr}(m, n)$
}

\author{
JAEHYUN HONG
}

\begin{abstract}
Let $\mathbf{a}=\left(p_{1}^{q_{1}}, \ldots, p_{r}^{q_{r}}\right)$ be a partition and $\mathbf{a}^{\prime}=\left(p_{1}^{\prime q_{1}^{\prime}}, \ldots, p_{r}^{\prime q_{r}^{\prime}}\right)$ be its conjugate. We will prove that if $q_{i}, q_{i}^{\prime} \geq 2$ for all $1 \leq i \leq r$, then any irreducible subvariety $X$ of $G r(m, n)$ whose homology class is an integral multiple of the Schubert class $\left[\sigma_{\mathbf{a}}\right]$ of type $\mathbf{a}$ is a Schubert variety of type $\mathbf{a}$.
\end{abstract}

\section{Introduction}

Let $\operatorname{Gr}(m, n)$ be the Grassmannian of $m$-planes in $\mathbb{C}^{n}$. For a partition $\mathbf{a}=\left(a_{1}, \ldots, a_{m}\right)$, a Schubert variety $\sigma_{\mathbf{a}}$ of type $\mathbf{a}$ is defined by the set of all $m$-planes $E$ such that $\operatorname{dim}\left(E \cap \mathbb{C}^{n-m+i-a_{i}}\right) \geq i$ for all $1 \leq i \leq m$ for a flag $\left\{\mathbb{C}^{1} \subset \ldots \subset \mathbb{C}^{n}\right\}$. Then they form a basis for the homology space $H_{*}(G r(m, n), \mathbb{Z})$.

For $\mathbf{a}=\left(p^{q}\right)^{*}=\left((n-m)^{m-q},(n-m-p)^{q}\right)$, the Schubert variety $\sigma_{\mathbf{a}}$ of type $\mathbf{a}$ is smooth and every smooth Schubert varieties in $\operatorname{Gr}(m, n)$ is of this form. Smooth Schubert varieties in $\operatorname{Gr}(m, n)$ are Schur rigid with trivial exceptions: for any smooth Schubert variety $\sigma_{\mathbf{a}}$ in $\operatorname{Gr}(m, n)$ other than a non-maximal linear space, any irreducible subvariety whose homology class is an integral multiple of the Schubert class $\left[\sigma_{\mathbf{a}}\right]$ of type $\mathbf{a}$ is a Schubert variety of type $\mathbf{a}([\mathbf{W}],[\mathbf{B}]$ and $[\mathbf{H o}])$.

In this paper, we will prove the Schur rigidity of singular Schubert varieties of certain types in $\operatorname{Gr}(m, n)$.

Theorem. Let $\mathbf{a}=\left(p_{1}^{q_{1}}, \ldots, p_{r}^{q_{r}}\right), p_{r} \neq 0$ be a partition and let $\mathbf{a}^{\prime}=$ $\left(p_{1}^{\prime} q_{1}^{\prime}, \ldots, p_{s}^{\prime} q_{r}^{\prime}\right), p_{r}^{\prime} \neq 0$ be its conjugate. Then, the Schubert variety $\sigma_{\mathbf{a}}$ in $\operatorname{Gr}(m, n)$ is Schur rigid if $q_{i}, q_{i}^{\prime} \geq 2$ for all $1 \leq i \leq r$.

The proof is divided by two parts as in $[\mathbf{W}],[\mathbf{B}]$ and $[\mathbf{H o}]$ : Schubert rigidity and the equality $\mathcal{B}_{\mathbf{a}}=\mathcal{R}_{\mathbf{a}^{*}}$. Schubert differential system $\mathcal{B}_{\mathbf{a}}$ is the differential system with a fiber at $x \in G r(m, n)$ given by the set of all the tangent space of Schubert varieties of type a passing through $x$. If any irreducible integral variety of $\mathcal{B}_{\mathbf{a}}$ is a Schubert variety of type $\mathbf{a}$, then we say that the Schubert variety $\sigma_{\mathbf{a}}$ is Schubert rigid.

Received 11/10/2004. 
Putting together the tangent space of all the subvarieties $X$ with $[X]=r\left[\sigma_{\mathbf{a}}\right], r \in \mathbb{Z}$ at each point, we get another differential system $\mathcal{R}_{\mathbf{a}^{*}}$, which we call the Schur differential system $([\mathbf{B}],[\mathbf{W}])$. By the construction, if any irreducible integral variety of $\mathcal{R}_{\mathbf{a}^{*}}$ is a Schubert variety of type a, then the Schubert variety $\sigma_{\mathbf{a}}$ is Schur rigid. Furthermore, the Schubert differential system $\mathcal{B}_{\mathbf{a}}$ is always contained in the Schur differential system $\mathcal{R}_{\mathbf{a}^{*}}$. Thus, the equality $\mathcal{B}_{\mathbf{a}}=\mathcal{R}_{\mathbf{a}^{*}}$ and the Schubert rigidity is a necessary and sufficient condition for the Schur rigidity $([\mathbf{B}])$. While proving the equality $\mathcal{B}_{\mathbf{a}}=\mathcal{R}_{\mathbf{a}^{*}}$ is computing integral elements of exterior differential systems, which is an algebraic problem, proving the Schubert rigidity is finding integral varieties of a differential system, which is a local differential geometric problem.

To prove the Schubert rigidity of a singular Schubert variety $\sigma_{\mathbf{a}}$, we use the fact that there is a natural foliation on $\sigma_{\mathbf{a}}$ by smooth Schubert varieties of type, say $\mathbf{b}$. But this does not say that any integral variety of $\mathcal{B}_{\mathbf{a}}$ is foliated by the Schubert varieties of type $\mathbf{b}$. We find a condition that is needed to get such a foliation by applying the theory on the integral varieties of $F$-structures by Goncharov (Section 3.2).

Once we get such a foliation on the integral variety of $\mathcal{B}_{\mathbf{a}}$, the space of leaves is again a Schubert variety of type, say $\mathbf{c}$, in the parameter space of the Schubert varieties of type $\mathbf{b}$. Then we can apply the induction to get the result. This inductive step forces us to consider the following problem: When $\sigma_{\mathbf{a}}$ is contained in a smooth Schubert variety of type $\mathbf{b}$, then will any integral variety of $\mathcal{B}_{\mathbf{a}}$ be contained in a Schubert variety of type $\mathbf{b}$ ? We obtain a condition which ensures such an inclusion by using the theory on the integral varieties of $F$-structures as above (Section 3.3).

To prove the equality $B_{\mathbf{a}}=R_{\mathbf{a}^{*}} \subset G r\left(k, E^{*} \otimes Q\right)$ for the fibers of $\mathcal{B}_{\mathbf{a}}$ and $\mathcal{R}_{\mathbf{a}^{*}}$ at $[E] \in G r(m, n)$, we use the description $R_{\mathbf{a}}=G r\left(k, E^{*} \otimes Q\right) \cap$ $\mathbb{P}\left(\mathbb{S}_{\mathbf{a}}\left(E^{*}\right) \otimes \mathbb{S}_{\mathbf{a}^{\prime}}(Q)\right)$ as in $[\mathbf{H o}]$ and then we compute the complement of the tangent space of $B_{\mathbf{a}}$ in the tangent space of $\operatorname{Gr}\left(k, E^{*} \otimes Q\right)$ by hands, while in $[\mathbf{H o}]$ the theory of Lie algebra cohomology developed by Kostant used to compute it (Section 4).

One of the applications covered in $[\mathbf{B}]$ is the one to the holomorphic vector bundles generated by global sections which satisfies vanishing of certain Chern classes. It is related to Schubert varieties in $\operatorname{Gr}(m, n)$ and from our result on the rigidity of Schubert varieties in $\operatorname{Gr}(m, n)$, one can get the classification of such holomorphic bundles for various vanishing conditions.

Even though Grassmannian $\operatorname{Gr}(m, n)$ we considered is a special Hermitian symmetric space, most of the method in this paper can be applied to prove the rigidity of singular Schubert varieties in general Hermitian symmetric space. The only thing to do is to verify the conditions in the above, which needs some works on the representation theory of simple 
Lie algebra. We expect that the result in this paper will be generalized to Hermitian symmetric spaces.

Acknowledgements. I wish to thank Professor D. Burns for suggesting the idea of using a natural foliation by smooth Schubert varieties on a singular Schubert variety to prove its rigidity. Some results of the present paper have been worked out while I was visiting MSRI in Berkeley in November 2003. I would like to thank this institute for its hospitality, and Professor R. Bryant, one of the organizers of the program "Differential Geometry", for stimulating discussions and encouragement. This work was supported by the Post-Doctorial Fellowship Program of Korea Science and Engineering Foundation (KOSEF).

\section{Differential systems}

2.1. Schubert and Schur differential systems. Let $\operatorname{Gr}(m, n)$ be the Grassmannian of $m$-dimensional subspaces of $V=\mathbb{C}^{n}$. Let $P(m, n)$ be the set

$$
\left\{\mathbf{a}=\left(a_{1}, \ldots, a_{m}\right) \mid n-m \geq a_{1} \geq \cdots \geq a_{m} \geq 0\right\}
$$

of partitions. Fix a flag $\left\{V_{\bullet}\right\}$ of $V$ with $\operatorname{dim} V_{i}=i$. For a $\in P(m, n)$, define the Schubert variety $\sigma_{\mathbf{a}}\left(V_{\bullet}\right)$ of type $\mathbf{a}$ by the set

$$
\left\{E \in G r(m, n) \mid \operatorname{dim}\left(E \cap V_{n-m+i-a_{i}}\right) \geq i\right\} .
$$

Then $\sigma_{\mathbf{a}}\left(V_{\bullet}\right)$ is an irreducible subvariety of $G r(m, n)$ of codimension $|\mathbf{a}|:=a_{1}+\cdots+a_{m}$. By varying the flag $\left\{V_{\bullet}\right\}$, we get a family of Schubert varieties $\sigma_{\mathbf{a}}$ of type $\mathbf{a}$.

For $\mathbf{a} \in P(m, n)$, define its dual $\mathbf{a}^{*}$ by

$$
\mathbf{a}^{*}=\left(n-m-a_{m}, \ldots, n-m-a_{1}\right)
$$

and define its conjugate $\mathbf{a}^{\prime}=\left(a_{1}^{\prime}, \ldots, a_{n-m}^{\prime}\right)$ by

$$
a_{i}^{\prime}=\text { the number of }\left\{j \mid a_{j} \geq i\right\} \text { for } 1 \leq i \leq n-m .
$$

The Young diagram $Y_{\mathbf{a}}$ is defined by the set of boxes consisting of $a_{i}$ boxes in the $i$-th row, the row of boxes lined up on the left.

Then the Young diagram $Y_{\mathbf{a}^{*}}$ is obtained by rotating the complement of the Young diagram $Y_{\mathbf{a}}$ by 180 degree and the Young diagram $Y_{\mathbf{a}^{\prime}}$ is obtained by transposing the Young diagram $Y_{\mathbf{a}}$.

Example. Let $m=5, n-m=6$. Young diagrams $Y_{\mathbf{a}}, Y_{\mathbf{a}^{*}}, Y_{\mathbf{a}^{\prime}}$ for $\mathbf{a}=(6,6,4,2,2) \cdot \mathbf{a}^{*}=(4,4,2), \mathbf{a}^{\prime}=(5,5,3,3,2,2)$ are given by 


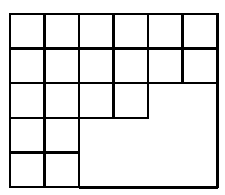

$Y_{\mathbf{a}}$

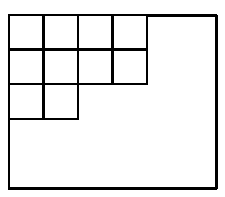

$Y_{\mathbf{a}^{*}}$

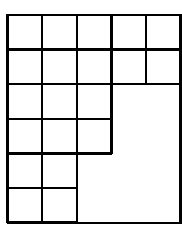

$Y_{\mathbf{a}^{\prime}}$

Define $\mathfrak{n}_{\mathbf{a}}$ to be the vector space of matrices $Z=\left(z_{i}^{a}\right) \in M_{n-m, m}$ that satisfy $z_{i}^{a}=0$ when $a>n-m-a_{i}$. This is the tangent space of the Schubert variety $\sigma_{\mathbf{a}}$.

For $\mathbf{a}$ as in the above Example, $\mathfrak{n}_{\mathbf{a}}$ is the spaces of all matrixes of the form

\begin{tabular}{|lllll|}
\hline 0 & 0 & $*$ & $*$ & $*$ \\
0 & 0 & $*$ & $*$ & $*$ \\
0 & 0 & 0 & $*$ & $*$ \\
0 & 0 & 0 & $*$ & $*$ \\
0 & 0 & 0 & 0 & 0 \\
0 & 0 & 0 & 0 & 0 \\
\hline
\end{tabular}

We will use the notation $\mathbf{a}=\left(p_{1}^{q_{1}}, \ldots, p_{r}^{q_{r}}\right), p_{r} \neq 0$ for the partition with $q_{1} p_{1}$ 's, $\ldots, q_{r} p_{r}$ 's.

Now, we will define two differential systems $\mathcal{B}_{\mathbf{a}}$ and $\mathcal{R}_{\mathbf{a}^{*}}$ associated to the Schubert variety $\sigma_{\mathbf{a}}$ following $[\mathbf{W}]$ and $[\mathbf{B}]$. A differential system $\mathcal{F}$ on a manifold $M$ is a subvariety of the Grassmannian bundle $\operatorname{Gr}(k, T M)$ of tangent $k$-subspaces of $M$. So, it assigns a family $\mathcal{F}_{x}$ of $k$-subspaces of the tangent space $T_{x} M$ at each point $x \in M$.

A subvariety $X$ of $M$ is said to be an integral variety of $\mathcal{F}$ if at each smooth point $x \in X$, its tangent space $T_{x} X$ is contained in the fiber $\mathcal{F}_{x}$. We say that $\mathcal{F}$ is integrable if at each point $x \in M$ and $y \in \mathcal{F}_{x}$, there is an integral variety passing through $x$ and tangent to the subspace $W_{y}$ of $T_{x} M$ corresponding to $y$.

A typical example of an integrable differential system is obtained from a family of subvarieties of $M$ which covers $M$, by assigning at each point $x \in M$ the set of all tangent space of the subvarieties in the family passing through $x$.

Definition. For each $\mathbf{a} \in P(m, n)$, the Schubert differential system $\mathcal{B}_{\mathbf{a}}$ of type $\mathbf{a}$ is the differential system with a fiber consisting of the tangent space to the Schubert varieties $\sigma_{\mathbf{a}}$ of type a passing through a given point. We say that $\sigma_{\mathbf{a}}$ is Schubert rigid if Schubert varieties of type a are the only irreducible integral varieties of $\mathcal{B}_{\mathbf{a}}$.

Let $[E] \in G r(m, n)$ and $Q=\mathbb{C}^{n} / E$. Then $T_{[E]} G r(m, n)=E^{*} \otimes Q$ and $\wedge^{k}\left(E^{*} \otimes Q\right)=\oplus_{|\mathbf{a}|=k} \mathbb{S}_{\mathbf{a}}\left(E^{*}\right) \otimes \mathbb{S}_{\mathbf{a}^{\prime}}(Q)$, where $\mathbb{S}_{\mathbf{a}}$ is the Schur functor of type a. There exists a $S U(n)$-invariant positive $(k, k)$-form $\phi_{\mathbf{a}}$ which 
can be written as the sum $(\sqrt{-1})^{k^{2}} \sum_{i} \xi_{i} \wedge \bar{\xi}_{i}$ at $[E]$, where $\left\{\xi_{i}\right\}$ is an orthonormal basis of $\mathbb{S}_{\mathbf{a}}(E) \otimes \mathbb{S}_{\mathbf{a}^{\prime}}\left(Q^{*}\right) \subset \wedge^{k}\left(T_{[E]}^{*} G r(m, n)\right)$. Then we have $\int_{\sigma_{\mathbf{a}^{*}}} \phi_{\mathbf{b}}=\delta_{\mathbf{a}}^{\mathbf{b}}$ for $\mathbf{a}, \mathbf{b} \in P(m, n)$. (For details, see $[\mathbf{B}]$.)

Definition. For each $\mathbf{a} \in P(m, n)$, the Schur differential system $\mathcal{R}_{\mathbf{a}}$ of type $\mathbf{a}$ is defined by the intersection

$$
\cap_{\mathbf{b} \neq \mathbf{a},|\mathbf{b}|=|\mathbf{a}|} Z\left(\phi_{\mathbf{b}}\right),
$$

where $Z\left(\phi_{\mathbf{b}}\right)$ is the set of $|b|$-subspace of $T_{E}(G r(m, n))$ on which $\phi_{\mathbf{b}}$ vanishes. We say that $\sigma_{\mathbf{a}}$ is Schur rigid if Schubert varieties of type $\mathbf{a}^{*}$ are the only integral varieties of $\mathcal{R}_{\mathbf{a}}$.

Thus $\mathcal{R}_{\mathbf{a}}$ is the differential system on $\operatorname{Gr}(m, n)$ such that $X$ is a subvariety of $\operatorname{Gr}(m, n)$ with $[X]=r\left[\sigma_{\mathbf{a}^{*}}\right]$ for an integer $r$ if and only if $X$ is an integral variety of $\mathcal{R}_{\mathbf{a}}([\mathbf{B}],[\mathbf{W}])$. Clearly, $\mathcal{B}_{\mathbf{a}^{*}}$ is contained in $\mathcal{R}_{\mathrm{a}}$.

2.2. $F$-structures, prolongations and integral varieties. In this section, we review the theory on the integral varieties of $F$-structures $([\mathbf{G}])$.

Definition. Let $F$ be a submanifold of $G r(k, V)$ with a transitive action of a subgroup of $G L(V)$. A fiber bundle $\mathcal{F} \subset G r(k, T M)$ on a manifold $M$ of dimension $n=\operatorname{dim} V$ is said to be an $F$-structure if at each point $x \in M$ there is a linear isomorphism $\varphi(x): V \rightarrow T_{x} M$ such that the induced map $\varphi(x)^{k}: G r(k, V) \rightarrow G r\left(k, T_{x} M\right)$ sends $F$ to $\mathcal{F}_{x}$.

Schubert differential systems are $F$-structures for various $F$ 's. Integrability and the uniqueness can be obtained by studying the cohomology space $H^{k, 1}(F)$ and $H^{k, 2}(F)$ associated to $\left(W_{f}, V, T_{f} F\right)$ for $f \in F([\mathbf{G}])$. When an $F$-structure is integrable, if $H^{k, 1}(F)=0$, then the family of all integral varieties passing through a fixed point and tangent to a fixed subspace has dimension $\sum_{j \leq k-1} \operatorname{dim} H^{j, 1}(F)$. In particular, if $H^{1,1}(F)=0$, there is only one such integral subvariety. Higher cohomology gives the information on the higher jet of the integral varieties.

In general, Schubert differential system for singular Schubert variety has order $\geq 2$, i.e. its integral varieties are determined by higher jets. But smooth Schubert varieties depend only 1-jet and there is a canonical map from the differential system of a singular Schubert variety to that of a certain smooth Schubert variety(Proposition 3.1). So, in this paper, we will consider only the first cohomology $H^{1,1}(F)$, which contains the information on the 2-jets of integral varieties.

Let $F$ be a subvariety of $\operatorname{Gr}(k, V)$ with a transitive action of a subgroup of $G L(V)$. Let $\mathcal{F}$ be an $F$-structure on $M$ with the projection map $\pi: \mathcal{F} \rightarrow M$. For $x \in M$ and $y \in \mathcal{F}_{x}$, let $W_{y}$ denote the $k$-subspace 
of $T_{x} M$ corresponding to $y$. For a $k$-subspace $H$ of $T_{y} \mathcal{F}$ such that $\pi_{*}: H \rightarrow W_{y}$ is an isomorphism, define $\partial H: \wedge^{2} W_{y} \rightarrow T_{x} M / W_{y}$ by

$$
\partial H\left(V_{1}, V_{2}\right)=\left[\tilde{V}_{1}, \tilde{V}_{2}\right] \bmod W_{y},
$$

$\tilde{V}_{i}, i=1,2$ is a local vector field on $M$ with $\tilde{V}_{i}\left(x^{\prime}\right) \in W_{\psi\left(x^{\prime}\right)}$ for a local section $\psi$ of $\mathcal{F}$ with $\psi_{*}\left(W_{y}\right)=H$. It is well defined $([\mathbf{G}])$.

A $k$-subspace $H$ of $T_{y} \mathcal{F}$ is said to be a 2-jet of an integral variety if $\pi_{*}$ restricts to an isomorphism $H \subset T_{y} \mathcal{F} \rightarrow W_{y} \subset T_{x} M$ and $\partial H=0$. Such an $H$ is indeed a candidate for the 2-jets of actual integral varieties of $\mathcal{F}$ and the set of such an $H$ is again a subvariety of $\operatorname{Gr}\left(k, V+T_{f} F\right)$.

Proposition 2.1. Let $\mathcal{F}$ be an F-structure on $M$. Let $x \in M$ and let $y \in \mathcal{F}_{x}$. If $X$ is an integral variety of $\mathcal{F}$ passing through $x$ and tangent to $W_{y}$, then the tangent space $H=T_{y} \tilde{X} \subset T_{y} \mathcal{F}$ of the lifting $\tilde{X}:=\left\{\left(x,\left[T_{x} X\right]\right) \mid x \in X,\left[T_{x} X\right] \in G r\left(k, T_{x} M\right)\right\}$ of $X$ to $G r\left(k, T_{x} M\right)$ satisfies

(1) $\pi_{*}: H \rightarrow W_{y}$ is an isomorphism

(2) $\partial H=0$.

Proof. See Chapter 1 of $[\mathbf{G}]$.

q.e.d.

The condition that $\pi_{*}: H \rightarrow W_{y}$ is an isomorphism is equivalent to the condition that $H$ is the graph of a map $p: W_{y} \rightarrow T_{y}\left(\mathcal{F}_{x}\right) \subset W_{y}^{*} \otimes$ $\left(T_{x} M / W_{y}\right)$. Define $\partial p: \wedge^{2} W_{y} \rightarrow T_{x} M / W_{y}$ by $\partial p\left(V_{1}, V_{2}\right)=p\left(V_{1}\right)\left(V_{2}\right)-$ $p\left(V_{2}\right)\left(V_{1}\right)$, considering $T_{y}\left(\mathcal{F}_{x}\right)$ as a subspace of $W_{y}^{*} \otimes\left(T_{x} M / W_{y}\right)$. Then, $\partial H=0$ if and only if $\partial p=0$.

Definition. Let $\mathcal{F}$ be an $F$-structure on $M$. The first prolongation $\mathcal{F}^{(1)}$ of $\mathcal{F}$ is defined by the union $\cup_{y \in \mathcal{F}} \mathcal{F}_{y}^{(1)}$ of the set of all 2-jets of integral varieties tangent to $W_{y}$ for $y \in \mathcal{F}$

Put $H^{1,1}(F)=\operatorname{Ker}\left(\partial: W_{f}^{*} \otimes T_{f} F \rightarrow \wedge^{2} W_{f}^{*} \otimes\left(V / W_{f}\right)\right)$ and put $F^{(1)}=\left\{H \subset V+T_{f} F \mid H\right.$ is the graph of a map $\left.p \in H^{1,1}(F)\right\}$, where $f \in F$. Then, $\mathcal{F}^{(1)}$ is an $F^{(1)}$-structure on $\mathcal{F}$.

If $H^{1,1}(F)=0$, then $F^{(1)}$ is just a point and the first prolongation defines a distribution on $\mathcal{F}$. In this case, the integrability of this distribution is equivalent to the integrability of the $F$-structure $\mathcal{F}$. So, there is at most one integral manifold passing through a given point and tangent to a given $k$-subspace of the tangent space. For the details, see Chapter 1 of $[\mathbf{G}]$.

\section{Schubert rigidity}

3.1. Description of the Schubert differential system. For a sequence $\left(n_{1}, \ldots, n_{r}\right)$ with $n_{1} \leq \cdots n_{r} \leq n$, denote by $F\left(n_{1}, \ldots, n_{r}, n\right)$ the flag space of all flags $\left(V_{1}, \ldots, V_{r}\right)$ in $V=\mathbb{C}^{n}$ with $\operatorname{dim} V_{i}=n_{i}$ for $1 \leq i \leq r$. 
Example. For $\mathbf{a}=\left(p^{q}\right)^{*}$, the Schubert variety $\sigma_{\mathbf{a}}$ of type $\mathbf{a}$ is the sub-Grassmannian $\left\{E \in G r(m, n) \mid \mathbb{C}^{m-q} \subset E \subset \mathbb{C}^{m+p}\right\} \simeq G r(q, p+q)$. The Schubert differential system $\mathcal{B}_{\mathbf{a}}$ is the flag space $F(m-q, m, m+$ $p, n)$ and the parameter space of the family of Schubert varieties of type $\mathbf{a}$ is $F(m-q, m+p, n)$. In this case, there is a double fibration $F(m-q, m+p, n) \leftarrow \mathcal{B}_{\mathbf{a}} \rightarrow G r(m, n)$.

In general, the Schubert differential systems $\mathcal{B}_{\mathbf{a}}$ is a generalized flag variety because $S L(n)$ acts on $\mathcal{B}_{\mathbf{a}}$ transitively and $\mathcal{B}_{\mathbf{a}}$ is compact. Thus, we have only to find the corresponding subset of simple root system generating the isotropy group which is parabolic.

Let $\mathcal{S}=\left\{\alpha_{1}, \ldots, \alpha_{n-1}\right\}$ be the set of simple roots of $G=S L(n)$ and let $P$ be the parabolic subgroup of $S L(n)$ generated by $\mathcal{S}^{1}=\mathcal{S}-\left\{\alpha_{m}\right\}$, i.e. $\mathcal{S}^{1}$ is the set of simple roots of the semisimple part $S L(m) \times S L(n-$ $m$ ) of $P$. Then, $G r(m, n)$ is expressed as $G / P$.

Proposition 3.1. For $\mathbf{a}=\left(p_{1}^{q_{1}}, \ldots, p_{r}^{q_{r}}\right), p_{r} \neq 0$, put

$$
\mathcal{S}_{\mathbf{a}}=\mathcal{S}^{1}-\left\{\alpha_{q_{1}}, \ldots, \alpha_{q_{1}+\cdots+q_{r}}\right\} \cup\left\{\alpha_{n-p_{1}} \ldots, \alpha_{n-p_{r}}\right\} .
$$

Let $Q_{\mathbf{a}}$ be the parabolic subgroup of $S L(n)$ generated by $\mathcal{S}_{\mathbf{a}} \cup\left\{\alpha_{m}\right\}$ and $P_{\mathbf{a}}$ be the parabolic subgroup of $S L(m) \times S L(n-m)$ generated by the set $\mathcal{S}_{\mathbf{a}}$. Then $\mathcal{B}_{\mathbf{a}}$ is the homogeneous manifold $G /\left(Q_{\mathbf{a}} \cap P\right)$ and $P_{\mathbf{a}}$ is the isotropy group of the action of $S L(m) \times S L(n-m)$ on the fiber $B_{\mathbf{a}}$ of $\mathcal{B}_{\mathbf{a}} \rightarrow G r(m, n)$. If $\mathcal{S}_{\mathbf{a}} \subset \mathcal{S}_{\mathbf{b}}$, then there exists a quotient map $\varphi_{\mathbf{a}, \mathbf{b}}: \mathcal{B}_{\mathbf{a}} \rightarrow \mathcal{B}_{\mathbf{b}}$ which preserves the fibers.

For example, the following figure describes $\mathfrak{n}_{\mathbf{a}}, \mathfrak{m}_{\mathbf{a}}$ and $Q_{\mathbf{a}}$ for $n=$ $10, m=4$ and $\mathbf{a}=(6,4,2,2)$, where $\mathfrak{m}_{\mathbf{a}}$ denotes the tangent space of $B_{\mathbf{a}} \cdot \mathfrak{n}_{\mathbf{a}}$ is the space of all $n \times n$-matrix with non-zero elements only in $*$ and $\mathfrak{m}_{\mathbf{a}}$ is the space of all $n \times n$-matrix with non-zero elements only in $\bullet$. The Lie algebra of the reductive part of $Q_{\mathbf{a}}$ is the space of all $n \times n$-matrix with non-zero elements only in $\diamond$.

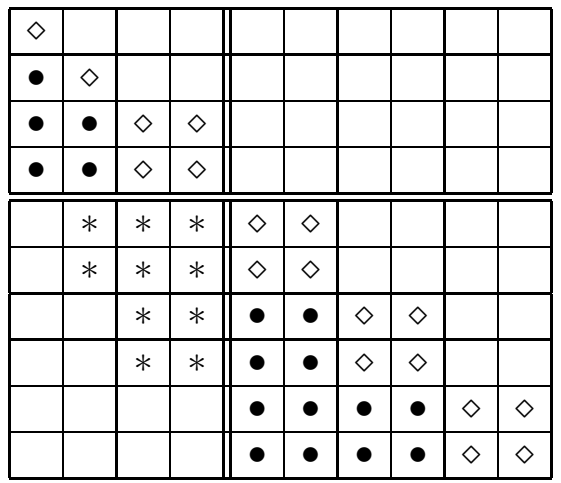


3.2. Foliation by smooth Schubert varieties. In general, a Schubert variety which is Schubert rigid depends on higher jets (See Example 13 in $[\mathbf{B}]$ and our Theorem). But smooth Schubert varieties in $\operatorname{Gr}(m, n)$ depends on only one jet, which will play a central role in proving the Schubert rigidity of singular Schubert varieties.

Proposition 3.2. For $\mathbf{a}=\left(p^{q}\right)^{*}$, the Schubert variety $\sigma_{\mathbf{a}}$ of type $\mathbf{a}$ is Schubert rigid except when $(p=1$ and $q \neq m)$ or $(p \neq n-m$ and $q=1)$. Furthermore, there is a unique Schubert variety passing through a given point and tangent to a given tangent subspace.

Proof. See $[\mathbf{W}]$ or $[\mathbf{H o}]$.

q.e.d.

We will say that a Schubert variety $\sigma_{\mathbf{a}}$ is strongly rigid if there is a unique Schubert variety passing through a given point and tangent to a given tangent subspace.

To prove the Schubert rigidity of general Schubert varieties, we start with the simplest case and then will use the induction to generalize it.

Example. The case when $\mathbf{a}=\left(p^{q}\right)$ is studied in Example 13 and Remark 33 of $[\mathbf{B}]$. Fix a $(n-m-p+q)$-subspace $\Lambda=\mathbb{C}^{n-m-p+q}$ of $\mathbb{C}^{n}$. Then, $\sigma_{\mathbf{a}}(\Lambda)$ can be expressed as a union of a family of Schubert varieties of type $\mathbf{b}=\left((n-m)^{q}\right)=\left((n-m)^{(m-q)}\right)^{*}$.

$$
\begin{aligned}
\sigma_{\mathbf{a}}(\Lambda) & =\{E \in G r(m, n) \mid \operatorname{dim}(E \cap \Lambda) \geq q\} \\
& =\bigcup_{\mathbb{C}^{q} \subset \Lambda}\left\{E \in G r(m, n) \mid \mathbb{C}^{q} \subset E\right\}
\end{aligned}
$$

Note that $\operatorname{Gr}(q, n)$ is the parameter space of the Schubert varieties of type $\mathbf{b}$ and $\left\{\mathbb{C}^{q} \in G r(q, n) \mid \mathbb{C}^{q} \subset \Lambda\right\}$ is a Schubert variety of type $\mathbf{c}=$ $\left((n-m-p)^{q}\right)^{*}$ in $\operatorname{Gr}(q, n)$.

$\mathbf{a}=$\begin{tabular}{|c|c||c|c|}
\hline 0 & 0 & 0 & 0 \\
\hline $\mathfrak{m}_{\mathbf{a}}$ & 0 & 0 & 0 \\
\hline \hline $\mathfrak{n}_{\mathbf{a}}$ & $\mathfrak{n}_{\mathbf{a}}$ & 0 & 0 \\
\hline 0 & $\mathfrak{n}_{\mathbf{a}}$ & $\mathfrak{m}_{\mathbf{a}}$ & 0 \\
\hline
\end{tabular}$\quad \mathbf{b}=$\begin{tabular}{|c|c||c|c|}
\hline 0 & 0 & 0 & 0 \\
\hline $\mathfrak{m}_{\mathbf{b}}$ & 0 & 0 & 0 \\
\hline 0 & $\mathfrak{n}_{\mathbf{b}}$ & 0 & 0 \\
\hline 0 & $\mathfrak{n}_{\mathbf{b}}$ & 0 & 0 \\
\hline
\end{tabular}$\quad \mathbf{c}=$\begin{tabular}{|c||c||c|c|c|}
\hline 0 & 0 & 0 & 0 \\
\hline 0 & $\mathfrak{m}_{\mathbf{c}}$ & 0 & 0 \\
\hline 0 & 0 & 0 & 0 \\
\hline
\end{tabular}

From this expression, we get the following desingularisation $\pi_{2}$ of $\sigma_{\mathbf{a}}(\Lambda)$.

$$
\begin{aligned}
& \mathcal{B}_{\mathbf{b}}=F(q, m, n) \stackrel{\pi_{1}}{\longrightarrow} \operatorname{Gr}(q, n) \supset G r(q, \Lambda) \\
& \quad \pi_{2} \downarrow \\
& \quad G r(m, n) \supset \sigma_{\mathbf{a}}(\Lambda)
\end{aligned}
$$

Here, $\sigma_{\mathbf{a}}(\Lambda)$ is equal to $\pi_{2}\left(\pi_{1}^{-1}(G r(q, \Lambda))\right)$ and $\pi_{2}: \pi_{1}^{-1}(G r(q, \Lambda)) \rightarrow$ $\sigma_{\mathbf{a}}(\Lambda)$ is generically one-to-one. The smooth locus of $\sigma_{\mathbf{a}}(\Lambda)$ is foliated by Schubert varieties of type $\mathbf{b}$ in $\operatorname{Gr}(m, n)$ and the space of leaves of this foliation is a Schubert variety of type $\mathbf{c}$ in $G r(q, n)$. 
In the same way as above, we get a different desingularisation of $\sigma_{\mathbf{a}}(\Lambda)$ by considering $\sigma_{\mathbf{a}}(\Lambda)$ as a union of a family of another type of Schubert varieties:

$$
\sigma_{\mathbf{a}}(\Lambda)=\bigcup_{\Lambda \subset \mathbb{C}^{n-p}}\left\{E \in G r(m, n) \mid E \subset \mathbb{C}^{n-p}\right\}
$$

Proposition 3.3. For the partition $\mathbf{a}=\left(p^{q}\right)$, the Schubert variety $\sigma_{\mathbf{a}}$ of type $\mathbf{a}$ is rigid if $p>1$ and $q>1$.

It is proved in Example 13 and Remark 33 of $[\mathbf{B}]$. We will prove it again in such a way that can be generalized to the case of the Schubert differential systems of other singular Schubert varieties.

Lemma 3.4. Let $\mathbf{a}$ and $\mathbf{b}$ be two partitions such that $\mathfrak{n}_{\mathbf{b}}$ is a subspace of $\mathfrak{n}_{\mathbf{a}}$ with $\mathcal{S}_{\mathbf{a}} \subset \mathcal{S}_{\mathbf{b}}$ (and thus, there is a projection $\varphi_{\mathbf{a}, \mathbf{b}}: \mathcal{B}_{\mathbf{a}} \rightarrow \mathcal{B}_{\mathbf{b}}$ as in Proposition 3.1 ). Assume that $\mathcal{B}_{\mathbf{b}}$ is strongly rigid. For $p: \mathfrak{n}_{\mathbf{a}} \rightarrow \mathfrak{m}_{\mathbf{a}}$, define $\tilde{p}$ by the composition $\mathfrak{n}_{\mathbf{a}} \rightarrow \mathfrak{m}_{\mathbf{a}} \rightarrow \mathfrak{m}_{\mathbf{b}}$.

If $\tilde{p}(v)=0$ for all $v \in \mathfrak{n}_{\mathbf{b}}$ and for all $p \in H^{1,1}\left(B_{\mathbf{a}}\right)$, then the smooth locus of any integral variety of $\mathcal{B}_{\mathbf{a}}$ is foliated by integral varieties of $\mathcal{B}_{\mathbf{b}}$, which are Schubert varieties of type $\mathbf{b}$.

Proof. By the strong rigidity of $\mathcal{B}_{\mathbf{b}}$, its first prolongation $\mathcal{B}_{\mathbf{b}}^{(1)}$ gives a distribution $D$ on $\mathcal{B}_{\mathrm{b}}$ which is integrable because $\mathcal{B}_{\mathrm{b}}$ is integrable. Integral varieties of $D$ are isomorphic to Schubert varieties of type $\mathbf{b}$ via the map $\pi_{\mathbf{b}}: \mathcal{B}_{\mathbf{b}} \rightarrow G r(m, n)$.

Let $X$ be an integral variety of $\mathcal{B}_{\mathbf{a}}$. Let $\tilde{X} \subset \mathcal{B}_{\mathbf{a}}$ be the lifting of $X$, that is, $\tilde{X}=\left\{\left(x,\left[T_{x} X\right]\right) \mid x \in X\right\}$. It suffices to show that $\varphi_{\mathbf{a}, \mathbf{b}}(\tilde{X})$ is foliated by the integral varieties of the distribution $D$ induced by $\mathcal{B}_{\mathrm{b}}^{(1)}$, that is, at each point $y \in \tilde{X},\left(\varphi_{\mathbf{a}, \mathbf{b}}\right)_{*}\left(T_{y} \tilde{X}\right)$ contains $D_{\varphi_{\mathbf{a}, \mathbf{b}}(y)}$.

$$
\begin{gathered}
\mathcal{B}_{\mathbf{b}}^{(1)} \\
\downarrow \\
\tilde{X} \subset \mathcal{B}_{\mathbf{a}} \stackrel{\varphi_{\mathbf{a}, \mathbf{b}}}{\longrightarrow} \mathcal{B}_{\mathbf{b}} \supset \varphi_{\mathbf{a}, \mathbf{b}}(\tilde{X}) \\
\searrow \swarrow \\
X \subset G r(m, n)
\end{gathered}
$$

The map $\left(\varphi_{\mathbf{a}, \mathbf{b}}\right)_{*}$ is given by the projection $\mathfrak{m}+\mathfrak{m}_{\mathbf{a}} \rightarrow \mathfrak{m}+\mathfrak{m}_{\mathbf{b}}$ and $T_{y} \tilde{X}$ is the graph of a map $p: \mathfrak{n}_{\mathbf{a}} \rightarrow \mathfrak{m}_{\mathbf{a}}$ with $\partial p=0$. So, $\left(\varphi_{\mathbf{a}, \mathbf{b}}\right)_{*}\left(T_{y} \tilde{X}\right)$ is the graph of the map $\mathfrak{n}_{\mathbf{a}} \stackrel{p}{\rightarrow} \mathfrak{m}_{\mathbf{a}} \rightarrow \mathfrak{m}_{\mathbf{b}}$ with $\partial p=0$. On the other hand, $D_{\varphi_{\mathbf{a}, \mathbf{b}}(y)}$ is the graph of the zero map $\mathfrak{n}_{\mathbf{b}} \rightarrow \mathfrak{m}_{\mathbf{b}}$. By the assumption, $\tilde{p}(v)$ is zero for all $v \in \mathfrak{n}_{\mathbf{b}}$, so $\varphi_{\mathbf{a}, \mathbf{b}}\left(T_{y} \tilde{X}\right)$ contains $D_{\varphi_{\mathbf{a}, \mathbf{b}}(y)}$. q.e.d.

Consider the Schubert differential system $\mathcal{B}_{\mathbf{a}}$ on $\operatorname{Gr}(m, n)$ for $\mathbf{a}=\left(p^{q}\right)$ and the Schubert differential system $\mathcal{B}_{\mathbf{c}}$ on $\operatorname{Gr}(q, n)$ for $\mathbf{c}=((n-m-$ $\left.p)^{q}\right)^{*}$. 
Lemma 3.5. For a subvariety $A \subset G r(q, n)$, define a subvariety $X_{A}$ of $G r(m, n)$ by $X_{A}:=\pi_{2}\left(\pi_{1}^{-1}(A)\right)$. If $X_{A}$ is an integral variety of $\mathcal{B}_{\mathbf{a}}$ on $\operatorname{Gr}(m, n)$ and $\operatorname{dim}\left(\pi_{1}^{-1}(A)\right)$ is equal to $\operatorname{dim}\left(X_{A}\right)$, then $A$ is an integral variety of $\mathcal{B}_{\mathbf{c}}$ on $\operatorname{Gr}(q, n)$.

$$
\begin{aligned}
& \quad \mathcal{B}_{\mathbf{b}}=F(q, m, n) \stackrel{\pi_{1}}{\longrightarrow} G r(q, n) \supset A \\
& \pi_{2} \downarrow \\
& \quad \operatorname{Gr}(m, n) \supset X_{A}
\end{aligned}
$$

Proof. We will follow the arguments in Example 16.6 of $[\mathbf{H}]$. Define $\tilde{\pi}_{i}, i=1,2$ to be the projection from $\operatorname{Gr}(q, n) \times G r(m, n)$ to the first and second component, respectively. Then $\pi_{2}\left(\pi_{1}^{-1}(A)\right)$ is $\tilde{\pi}_{2}\left(\tilde{\pi}_{1}^{-1}(A) \cap\right.$ $F(q, m, n))$.

Let $\Gamma$ be a smooth point in $A$. Then $\tilde{\pi}_{1}^{-1}(A)$ is smooth at $(\Gamma, E)$ for all $E \in G r(m, n)$ with the tangent space

$$
T_{(\Gamma, E)} \tilde{\pi}_{1}^{-1}(A)=\left\{(\eta, \varphi) \mid \begin{array}{l}
\eta: \Gamma \rightarrow \mathbb{C}^{n} / \Gamma, \\
\varphi: E \rightarrow \mathbb{C}^{n} / E,
\end{array} \quad \eta \in T_{\Gamma} A\right\} .
$$

The tangent space of $F(q, m, n)$ at $(\Gamma, E)$ is

$$
T_{(\Gamma, E)} F(q, m, n)=\left\{(\eta, \varphi) \mid \begin{array}{l}
\eta: \Gamma \rightarrow \mathbb{C}^{n} / \Gamma, \\
\varphi: E \rightarrow \mathbb{C}^{n} / E,\left.\quad \varphi\right|_{\Gamma} \equiv \eta \bmod E
\end{array}\right\} .
$$

By dimension counting, we see that the two tangent spaces are transversal so that $\pi_{1}^{-1}(A)=\tilde{\pi}_{1}^{-1}(A) \cap F(q, m, n)$ is smooth at all $(\Gamma, E)$ with $\Gamma \subset E$, and the tangent space of $\pi_{1}^{-1}(A)$ at $(\Gamma, E)$ is given by

$$
T_{(\Gamma, E)} \pi_{1}^{-1}(A)=\left\{(\eta, \varphi) \mid \begin{array}{l}
\eta: \Gamma \rightarrow \mathbb{C}^{n} / \Gamma, \quad \eta \in T_{\Gamma} A \\
\varphi: E \rightarrow \mathbb{C}^{n} / E,\left.\quad \varphi\right|_{\Gamma} \equiv \eta \quad \bmod E
\end{array}\right\} .
$$

For each $E \in X_{A}$, there are only finitely many $\Gamma \in A$ with $\Gamma \subset E$. If there are more than one $\Gamma \in A$ with $\Gamma \subset E$, then $X_{A}$ is not smooth at $E$ (Proposition 16.8 of $[\mathbf{H}]$ ). Let $E$ be an element in $X_{A}$ such that there is only one $\Gamma \in A$ with $\Gamma \subset E$. Then, $\pi_{2}: \pi_{1}^{-1}(A) \rightarrow X_{A}$ is one-to-one over $E$ so $X_{A}$ is smooth at $E$ with the tangent space

$$
T_{E}\left(X_{A}\right)=\left\{\varphi: E \rightarrow \mathbb{C}^{n} / E|\varphi|_{\Gamma} \equiv \eta \quad \bmod E, \eta \in T_{\Gamma} A,\right\} .
$$

Since $T_{E}\left(X_{A}\right)$ is of type $\mathbf{a}$ in $\operatorname{Gr}(m, n), T_{\Gamma} A$ is of type $\mathbf{c}$ in $G r(q, n)$.

q.e.d.

Proof of Proposition 3.3. We will show that for all $p: \mathfrak{n}_{\mathbf{a}} \rightarrow \mathfrak{m}_{\mathbf{a}}$ with $p \in H^{1,1}\left(B_{\mathbf{a}}\right)$, the induced map $\tilde{p}: \mathfrak{n}_{\mathbf{b}} \subset \mathfrak{n}_{\mathbf{a}} \rightarrow \mathfrak{m}_{\mathbf{a}} \rightarrow \mathfrak{m}_{\mathbf{b}}$ is zero. Then, for an integral variety $X$ of $\mathcal{B}_{\mathbf{a}}$, the space of leaves $A=\pi_{1}\left(\varphi_{\mathbf{a}, \mathbf{b}}(\tilde{X})\right) \subset$ $\operatorname{Gr}(q, n)$ of the foliation on $X$ given by Lemma 3.4 satisfies the conditions in Lemma 3.5. Thus, $A \subset G r(q, n)$ is an integral variety of $\mathcal{B}_{\mathbf{c}}(G r(q, n))$. Since $q>1 \mathcal{B}_{\mathbf{c}}$ is rigid. So, $A$ is the Schubert variety $\operatorname{Gr}(q, \Lambda)$ for a 
$(n-m-p+q)$-subspace $\Lambda$ of $\mathbb{C}^{n}$ and hence, $X$ is the Schubert variety $\sigma_{\mathbf{a}}(\Lambda)$.

Let $E_{i, j}$ be the $n \times n$-matrix with only one non-zero element in the $i$-th row and $j$-th column. Then, $\left[E_{i, j}, E_{k, \ell}\right]=\delta_{j, k} E_{i \ell}-\delta_{i, \ell} E_{k, j}$ for all $1 \leq i, j, k, \ell \leq n$.

Since $[p(X), Y]-[p(Y), X] \in \mathfrak{n}_{\mathbf{a}}$ for all $X, Y \in \mathfrak{n}_{\mathbf{a}}$ and $\left[\mathfrak{m}_{\mathbf{a}} / \mathfrak{m}_{\mathbf{b}}, \mathfrak{n}_{\mathbf{b}}\right] \subset$ $\mathfrak{n}_{\mathbf{b}},[\tilde{p}(X), Y]-[\tilde{p}(Y), X] \in \mathfrak{n}_{\mathbf{a}}$ for all $X, Y \in \mathfrak{n}_{\mathbf{b}}$. Assume that $q+1 \leq$ $i, j \leq m$ and $m+1 \leq r, s \leq n-p$ and $n-p+1 \leq a, b \leq n$. Note that for a fixed $a$, if $X \in \mathfrak{m}_{\mathbf{b}}$ and $\left[X, E_{a, i}\right]=0$ for all $i$, then $X=0$.

\begin{tabular}{|c|c||c|c|}
\hline 0 & 0 & 0 & 0 \\
\hline $\mathfrak{m}_{\mathbf{b}}$ & 0 & 0 & 0 \\
\hline \hline$*$ & $E_{r, i}$ & 0 & 0 \\
\hline 0 & $E_{a, i}$ & $\bullet$ & 0 \\
\hline
\end{tabular}

From $\left[\tilde{p}\left(E_{a, i}\right), E_{r, j}\right]-\left[\tilde{p}\left(E_{r, j}\right), E_{a, i}\right] \in \mathfrak{n}_{\mathbf{a}}$, we get both $\left[\tilde{p}\left(E_{a, i}\right), E_{r, j}\right]$ and $\left[\tilde{p}\left(E_{r, j}\right), E_{a, i}\right]$ are contained in $\mathfrak{n}_{\mathbf{a}}$. Thus, $\left[\tilde{p}\left(E_{r, j}\right), E_{a, i}\right]$ is zero, which implies that $\tilde{p}\left(E_{r, j}\right)=0$.

Since $\left[\tilde{p}\left(E_{a, i}\right), E_{b, j}\right]-\left[\tilde{p}\left(E_{b, j}\right), E_{a, i}\right]$ is contained in $\mathfrak{n}_{\mathbf{a}}$, it should be zero and thus, both $\left[\tilde{p}\left(E_{a, i}\right), E_{b, j}\right]$ and $\left[\tilde{p}\left(E_{b, j}\right), E_{a, i}\right]$ should be zero for $a \neq b$. Here, we use the condition that $p>1$. So, $\tilde{p}\left(E_{b, j}\right)$ is zero. q.e.d.

3.3. Sub-Grassmannians. To extend the result in Proposition 3.3 to the general case, we consider the following problem: Suppose that a Schubert variety $\sigma_{\mathbf{a}}$ is contained in a proper sub-Grassmannian of $\operatorname{Gr}(m, n)$ and $\sigma_{\mathbf{b}}$ is the minimal sub-Grassmannian among them. Then, will any integral variety of $\mathcal{B}_{\mathbf{a}}$ be contained in a sub-Grassmannian $\sigma_{\mathbf{b}}$ ?

Proposition 3.6. Let $\mathbf{a}=\left(p_{1}^{q_{1}}, \ldots, p_{r}^{q_{r}}\right) \in P(m, n)$ be a partition and let $\mathbf{a}^{\prime}=\left(p_{1}^{\prime q_{1}^{\prime}}, \ldots, p_{r}^{\prime q_{r}^{\prime}}\right)$ be the conjugate of $\mathbf{a}$. Suppose that $\mathfrak{n}_{\mathbf{a}}$ is contained in a proper rectangle in $\mathbf{m}$. Let $\mathbf{b}$ be the partition corresponding to the minimal rectangle among them. Then any integral variety of $\mathcal{B}_{\mathbf{a}}$ is contained in a sub-Grassmannian $\sigma_{\mathbf{b}}$ except when $q_{1}+\cdots+q_{r}=m$ and $q_{r}=1$ or $q_{1}^{\prime}+\cdots+q_{r}^{\prime}=n-m$ and $q_{r}^{\prime}=1$.

If both $q_{1}+\cdots+q_{r}<m$ and $q_{1}^{\prime}+\cdots+q_{r}^{\prime}<n-m$ hold, then there is no proper sub-Grassmnnian containing $\sigma_{\mathbf{a}}$. So, the cases we will consider below are either when $q_{1}+\cdots+q_{r}=m$ and $q_{r} \geq 2$ or when $q_{1}^{\prime}+\cdots+q_{r}^{\prime}=n-m$ and $q_{r}^{\prime} \geq 2$.

Lemma 3.7. Let $\mathbf{a}$ and $\mathbf{b}$ be two partitions such that $\mathfrak{n}_{\mathbf{a}}$ is a subspace of $\mathfrak{n}_{\mathbf{b}}$ with $\mathcal{S}_{\mathbf{a}} \subset \mathcal{S}_{\mathbf{b}}$ and thus, there is a projection $\mathcal{B}_{\mathbf{a}} \rightarrow \mathcal{B}_{\mathbf{b}}$ as in Proposition 3.1. Assume that $\mathcal{B}_{\mathbf{b}}$ is strongly rigid. For $p: \mathfrak{n}_{\mathbf{a}} \rightarrow \mathfrak{m}_{\mathbf{a}}$, define $\tilde{p}$ by the composite map $\mathfrak{n}_{\mathbf{a}} \rightarrow \mathfrak{m}_{\mathbf{a}} \rightarrow \mathfrak{m}_{\mathbf{b}}$. If $\tilde{p}=0$ for all $p \in$ $H^{1,1}\left(B_{\mathbf{a}}\right)$, then any integral subvariety of $\mathcal{B}_{\mathbf{a}}$ is contained in an integral variety of $\mathcal{B}_{\mathbf{b}}$, which is a Schubert variety of type $\mathbf{b}$. 
Proof. The proof is similar to the proof of Lemma 3.4 q.e.d.

Proof of Proposition 3.6. First, we consider the case $\mathbf{a}=\left(p_{1}^{q_{1}}, p_{2}^{q_{2}}, p_{3}^{q_{3}}\right)$, $p_{3} \neq 0$ is a partition with $q_{1}+q_{2}+q_{3}=m$ and $q_{3} \geq 2$. As the proof will show, the general case can be obtained in the same way.

Claim. Put $\mathbf{b}=\left(p_{3}^{m}\right)$.

$$
\mathbf{a}=\begin{array}{|c|c|c||c|c|c|}
\hline 0 & 0 & 0 & 0 & 0 & 0 \\
\hline \bullet & 0 & 0 & 0 & 0 & 0 \\
\hline \bullet & \bullet & 0 & 0 & 0 & 0 \\
\hline \hline 0 & * & * & 0 & 0 & 0 \\
\hline 0 & 0 & * & \bullet & 0 & 0 \\
\hline 0 & 0 & 0 & \bullet & \bullet & 0 \\
\hline
\end{array} \quad \mathbf{b}=\begin{array}{|c|c|c|c||c|c|c|}
\hline 0 & 0 & 0 & 0 & 0 & 0 \\
\hline 0 & 0 & 0 & 0 & 0 & 0 \\
\hline 0 & 0 & 0 & 0 & 0 & 0 \\
\hline \hline * & * & * & 0 & 0 & 0 \\
\hline * & * & * & 0 & 0 & 0 \\
\hline 0 & 0 & 0 & \bullet & \bullet & 0 \\
\hline
\end{array}
$$

Then any integral variety of $\mathcal{B}_{\mathbf{a}}$ is contained in a Schubert variety of type $\mathbf{b}$.

Proof of the Claim. Let $p: \mathfrak{n}_{\mathbf{a}} \rightarrow \mathfrak{m}_{\mathbf{a}}$ be a map with $\partial p=0$ Then $[p(X), Y]-[p(Y), X] \in \mathfrak{n}_{\mathbf{a}}$ for all $X, Y \in \mathfrak{n}_{\mathbf{a}}$. Put $\tilde{p}: \mathfrak{n}_{\mathbf{a}} \rightarrow \mathfrak{m}_{\mathbf{b}}$ to be the composition of $p$ with the projection $\mathfrak{m}_{\mathbf{a}} \rightarrow \mathfrak{m}_{\mathbf{b}}$.

Since $\left[\mathfrak{m}_{\mathbf{a}} / \mathfrak{m}_{\mathbf{b}}, \mathfrak{n}_{\mathbf{a}}\right] \subset \mathfrak{n}_{\mathbf{b}}$, we have $[\tilde{p}(X), Y]-[\tilde{p}(Y), X] \in \mathfrak{n}_{\mathbf{b}}$ for all $X, Y \in \mathfrak{n}_{\mathbf{a}}$. Assume that $q_{1}+1 \leq k, \ell \leq q_{1}+q_{2}, q_{1}+q_{2}+1 \leq i, j \leq m$, $m+1 \leq r, s \leq n-p_{1}$ and $n-p_{1}+1 \leq a, b \leq n-p_{2}$. Note that for $X \in \mathfrak{m}_{\mathbf{b}}$, if $\left[X, E_{r, i}\right]=\left[X, E_{a, i}\right]=0$ for all $r$ and $a$ for a fixed $i$, then $X=0$.

\begin{tabular}{|c|c|c||c|c|c|}
\hline 0 & 0 & 0 & 0 & 0 & 0 \\
\hline$\bullet$ & 0 & 0 & 0 & 0 & 0 \\
\hline$\bullet$ & $\bullet$ & 0 & 0 & 0 & 0 \\
\hline \hline 0 & $E_{r, k}$ & $E_{r, i}$ & 0 & 0 & 0 \\
\hline 0 & 0 & $E_{a, i}$ & $\bullet$ & 0 & 0 \\
\hline 0 & 0 & 0 & $\mathfrak{m}_{\mathbf{b}}$ & $\mathfrak{m}_{\mathbf{b}}$ & 0 \\
\hline
\end{tabular}

From $\left[\tilde{p}\left(E_{r, k}\right), E_{s, i}\right]-\left[\tilde{p}\left(E_{s, i}\right), E_{r, k}\right] \in \mathfrak{n}_{\mathbf{b}}$, we see that both $\left[\tilde{p}\left(E_{r, k}\right), E_{s, i}\right]$ and $\left[\tilde{p}\left(E_{s, i}\right), E_{r, k}\right]$ are contained in $\mathfrak{n}_{\mathbf{b}}$. So, $\left[\tilde{p}\left(E_{r, k}\right), E_{s, i}\right]=0$. The same equation holds if we replace $E_{s, i}$ by $E_{a, i}$. Thus, $\tilde{p}\left(E_{r, k}\right)=0$.

Put $\mathbf{c}=\left((n-m)^{\left(q_{1}+q_{2}\right)}, p_{3}^{q_{3}}\right)$.

$$
\mathbf{c}=\begin{array}{|l|l|l||l|l|l|}
\hline 0 & 0 & 0 & 0 & 0 & 0 \\
\hline 0 & 0 & 0 & 0 & 0 & 0 \\
\hline \bullet & \bullet & 0 & 0 & 0 & 0 \\
\hline \hline 0 & 0 & * & 0 & 0 & 0 \\
\hline 0 & 0 & * & 0 & 0 & 0 \\
\hline 0 & 0 & 0 & \bullet & \bullet & 0 \\
\hline
\end{array}
$$


Then $\tilde{p}$ restricts to a map $\mathfrak{n}_{\mathbf{c}} \rightarrow \mathfrak{m}_{\mathbf{b}} \subset \mathfrak{m}_{\mathbf{c}}$. Also, we have $[\tilde{p}(X), Y]-$ $[\tilde{p}(Y), X] \in \mathfrak{n}_{\mathbf{c}}$ for all $X, Y \in \mathfrak{n}_{\mathbf{c}}$. Since $q_{3} \geq 2, \mathcal{B}_{\mathbf{c}}$ is strongly rigid so that $\tilde{p}$ is zero on $\mathfrak{n}_{\mathbf{c}}$. Hence, $\tilde{p}$ is zero. By Lemma 3.7, any integral variety of $\mathcal{B}_{\mathbf{a}}$ is contained in a Schubert variety of type $\mathbf{b}$.

In the same way, we can show that any integral manifold of $\mathcal{B}_{\mathbf{a}}$ is contained in a Schubert variety of type $\hat{\mathbf{b}}$ for $\hat{\mathbf{b}}=\left(p_{3}^{\prime n-m}\right)$.

$$
\mathbf{a}=\begin{array}{|c|c|c||c|c|c|}
\hline 0 & 0 & 0 & 0 & 0 & 0 \\
\hline \bullet & 0 & 0 & 0 & 0 & 0 \\
\hline \bullet & \bullet & 0 & 0 & 0 & 0 \\
\hline \hline 0 & * & * & 0 & 0 & 0 \\
\hline 0 & 0 & * & \bullet & 0 & 0 \\
\hline 0 & 0 & 0 & \bullet & \bullet & 0 \\
\hline
\end{array}
$$

$\hat{\mathbf{b}}=$\begin{tabular}{|c|c|c||c|c|c|}
\hline 0 & 0 & 0 & 0 & 0 & 0 \\
\hline$\bullet$ & 0 & 0 & 0 & 0 & 0 \\
\hline$\bullet$ & 0 & 0 & 0 & 0 & 0 \\
\hline \hline 0 & $*$ & $*$ & 0 & 0 & 0 \\
\hline 0 & $*$ & $*$ & 0 & 0 & 0 \\
\hline 0 & $*$ & $*$ & 0 & 0 & 0 \\
\hline
\end{tabular}

Here, we use the rigidity of the Schubert differential system $\mathcal{B}_{\hat{\mathbf{c}}}$ with $\hat{\mathbf{c}}=\left(m^{\left(q_{1}^{\prime}+q_{2}^{\prime}\right)}, p_{3}^{\prime}{ }_{3}^{\prime}\right), q_{3}^{\prime} \geq 2$.

$$
\hat{\mathbf{c}}=\begin{array}{|l|l|l||l|l|l|}
\hline 0 & 0 & 0 & 0 & 0 & 0 \\
\hline \bullet & 0 & 0 & 0 & 0 & 0 \\
\hline \bullet & 0 & 0 & 0 & 0 & 0 \\
\hline \hline 0 & * & * & 0 & 0 & 0 \\
\hline 0 & 0 & 0 & \bullet & 0 & 0 \\
\hline 0 & 0 & 0 & \bullet & 0 & 0 \\
\hline
\end{array}
$$

q.e.d.

Theorem 3.8. Let $\mathbf{a}=\left(p_{1}^{q_{1}}, \ldots, p_{r}^{q_{r}}\right)$ be a partition and let $\mathbf{a}^{\prime}=$ $\left(p_{1}^{\prime q_{1}^{\prime}}, \ldots, p_{r}^{\prime} q_{r}^{\prime}\right)$ be its conjugate. Then, $\sigma_{\mathbf{a}}$ is Schubert rigid if $q_{i}, q_{i}^{\prime} \geq 2$ for all $i \leq r$.

Proof. We will use the induction on $r$. Thanks to Proposition 3.6, we may assume that there is no proper sub-Grassmannian containing $\sigma_{\mathbf{a}}$ so that $q_{1}+\cdots q_{r}<m$ and $q_{1}^{\prime}+\cdots q_{r}^{\prime}<n-m$.

By Lemma 3.4 and the same argument as in Proposition 3.3, any integral varieties of $\mathcal{B}_{\mathbf{a}}$ are foliated by the Schubert varieties of type $\mathbf{b}=\left((n-m)^{q}\right)$, where $q=q_{1}+\cdots q_{r}$. Then the space of leaves $A$ of this foliation will be an integral variety of the Schubert differential system $\mathcal{B}_{\mathbf{c}}$ on $\operatorname{Gr}(q, n), \mathbf{c}=\left(\left(p_{1}+(m-q)\right)^{q_{1}}, \ldots,\left(p_{r}+(m-q)\right)^{q_{r}}\right)$. By Proposition 3.6, any integral varieties of $\mathcal{B}_{\mathbf{c}}$ are contained in a subGrassmannian of type $\left(\left(p_{r}+(m-q)\right)^{q}\right)$ in $\operatorname{Gr}(q, n)$. Thus, $A$ is an integral variety of the Schubert differential system $\mathcal{B}_{\mathbf{d}}, \mathbf{d}=\left(\left(p_{1}-\right.\right.$ $\left.\left.p_{r}\right)^{q_{1}}, \ldots,\left(p_{r-1}-p_{r}\right)^{q_{r-1}}\right)$ on this sub-Grassmannian, which is rigid by the induction hypothesis.

q.e.d. 


\section{Schur rigidity}

Let $\sigma_{\mathbf{a}}$ be a Schubert variety of type $\mathbf{a}$ in $\operatorname{Gr}(m, n)$. If $\sigma_{\mathbf{a}}$ is Schubert rigid and the Schur differential system $\mathcal{R}_{\mathbf{a}^{*}}$ is equal to the Schubert differential system $\mathcal{B}_{\mathbf{a}}$, then $\sigma_{\mathbf{a}}$ is Schur rigid. In the previous section, we proved the Schubert rigidity of $\sigma_{\mathbf{a}}$ under some assumptions on a (Theorem 3.8). In this section, we will prove that $\mathcal{B}_{\mathbf{a}}$ is equal to $\mathcal{R}_{\mathbf{a}^{*}}$ under a weaker assumption (Proposition 4.3) and that this assumption is critical (Remark 2).

4.1. Criterions for the equality $B_{\mathbf{a}}=R_{\mathbf{a}^{*}}$. Let $\mathbf{a}$ be a partition in $P(m, n)$ and $\mathbf{b}$ be a partition with $|\mathbf{b}|=|\mathbf{a}|$. Since $\phi_{\mathbf{b}}$ is equal to $\left.(\sqrt{-1})^{\mid \mathbf{b}}\right|^{2} \sum_{i} \xi_{i} \wedge \bar{\xi}_{i}$ for an orthonormal basis $\left\{\xi_{i}\right\}$ of $\left(\mathbb{S}_{\mathbf{b}}\left(E^{*}\right) \otimes\right.$ $\left.\mathbb{S}_{\mathbf{b}^{\prime}}(Q)\right)^{*}$ at the origin, the intersection $R_{\mathbf{a}}=\cap_{\mathbf{b} \neq \mathbf{a},|\mathbf{b}|=|\mathbf{a}|} Z\left(\phi_{\mathbf{b}}\right)$ is equal to $G r\left(|\mathbf{a}|, E^{*} \otimes Q\right) \cap \mathbb{P}\left(\mathbb{S}_{\mathbf{a}}\left(E^{*}\right) \otimes \mathbb{S}_{\mathbf{a}^{\prime}}(Q)\right) \subset \mathbb{P}\left(\wedge^{|\mathbf{a}|}\left(E^{*} \otimes Q\right)\right.$ ) (Proposition 2.8 of $[\mathbf{H o}])$.

Note that we adapt the convention that $\sigma_{\mathbf{a}}$ is a Schubert variety of dimension $\left|\mathbf{a}^{*}\right|$, while $X_{w}$ is a Schubert variety of dimension $\ell(w)$ in [Ho]. Thus, when $\mathbf{a}^{*} \in P(m, n)$ corresponds to $w \in W^{P}, \mathcal{B}_{\mathbf{a}}$ is equal to $\mathcal{B}_{w}$ and $\mathcal{R}_{\mathbf{a}^{*}}$ is equal to $\mathcal{R}_{w}$.

From the transitive action of $P_{0}$ on the fiber $B_{\mathbf{a}}$, we have the decomposition $\mathfrak{p}_{0}=\mathfrak{m}_{\mathbf{a}}+\mathfrak{l}_{\mathbf{a}}+\mathfrak{m}_{\mathbf{a}}^{*}$, where the tangent space of $B_{\mathbf{a}}$ at a point is isomorphic to $\mathfrak{m}_{\mathbf{a}}$. Put $\mathbf{I}_{\mathbf{a}}=\mathbb{S}_{\mathbf{a}^{*}}\left(E^{*}\right) \otimes \mathbb{S}_{\left(\mathbf{a}^{*}\right)^{\prime}}(Q)$. By comparing the tangent space $T_{\left[\mathfrak{n}_{\mathbf{a}}\right]} B_{\mathbf{a}}$ and the intersection of the tangent spaces $T_{\left[\mathfrak{n}_{\mathbf{a}}\right]} G r\left(k, E^{*} \otimes Q\right)$ and $T_{\left[\wedge \mathfrak{n}_{\mathbf{a}}\right]} \mathbb{P}\left(\mathbf{I}_{\mathbf{a}}\right)$, we get a sufficient condition for the equality $B_{\mathbf{a}}=R_{\mathbf{a}^{*}}$ as Proposition 3.4 in $[\mathbf{H o}]$.

Proposition 4.1. Assume that for the highest weight vector $\varphi$ of every irreducible $\mathfrak{l}_{\mathbf{a}}$-representation space in the complement of $\mathfrak{m}_{\mathbf{a}}$ in $\mathfrak{n}_{\mathbf{a}}^{*} \otimes \mathfrak{m} / \mathfrak{n}_{\mathbf{a}}$, we have

$$
\varphi^{k}\left(v_{1} \wedge \cdots \wedge v_{k}\right) \notin \mathbf{I}_{\mathbf{a}} / \wedge^{k} \mathfrak{n}_{\mathbf{a}},
$$

where $\left\{v_{1}, \ldots, v_{k}\right\}$ is a basis of $\mathfrak{n}_{\mathbf{a}}$ and $\varphi^{k}: \wedge^{k} \mathfrak{n}_{\mathbf{a}} \rightarrow \wedge^{k} \mathfrak{m} / \wedge^{k} \mathfrak{n}_{\mathbf{a}}$ is defined by

$$
\varphi^{k}\left(v_{1} \wedge \cdots \wedge v_{k}\right)=\sum_{i} v_{1} \wedge \cdots \wedge \varphi\left(v_{i}\right) \wedge \cdots \wedge v_{k} \quad \bmod \wedge^{k} \mathfrak{n}_{\mathbf{a}}
$$

Then $B_{\mathbf{a}}$ is equal to $R_{\mathbf{a}^{*}}$.

Now, we will compute all the irreducible $\mathfrak{l}_{\mathbf{a}}$-representation spaces in the complement of $\mathfrak{m}_{\mathbf{a}}$ in $\mathfrak{n}_{\mathbf{a}}^{*} \otimes \mathfrak{m} / \mathfrak{n}_{\mathbf{a}}$. Let $E$ be an $m$-subspace of $\mathbb{C}^{n}$ and $Q$ be the quotient $\mathbb{C}^{n} / E$. Then $\mathfrak{m}$ is equal to $E^{*} \otimes Q$. Take a partition $\mathbf{a}=\left(p_{1}^{q_{1}}, \ldots, p_{r}^{q_{r}}\right) \in P(m, n)$ and let $\mathbf{a}^{\prime}=\left(p_{1}^{\prime}{ }_{1}^{\prime}, \ldots, p_{r}^{\prime}{ }_{r}^{\prime}\right), p_{r}^{\prime} \neq 0$ be its conjugate.

Write $E=\oplus_{i=1}^{r_{E}} E_{i}$ and $Q=\oplus_{a=1}^{r_{Q}} Q_{a}$ so that $\mathfrak{l}_{\mathbf{a}}=\left(\oplus_{i=1}^{r_{E}} s l\left(E_{i}\right)\right) \oplus$ $\left(\oplus_{a=1}^{r_{Q}} \operatorname{sl}\left(Q_{a}\right)\right)$. Note that $r_{E}$ is $r$ if $q_{1}+\cdots+q_{r}=m$ and is $r+1$, 
otherwise, and $r_{Q}$ is $r$ if $q_{1}^{\prime}+\cdots q_{r}^{\prime}=n-m$ and is $r+1$, otherwise. We indexed $E_{i}$ and $Q_{a}$ keeping the order of the basis $\left\{e_{1}, \ldots, e_{n}\right\}$ of $\mathbb{C}^{n}$ such that $\left\{e_{1}, \ldots, e_{m}\right\}$ is a basis of $E$. Set $r_{i}=\operatorname{dim} E_{i}$ and $s_{a}=\operatorname{dim} Q_{a}$ for $1 \leq i \leq r_{E}$ and $1 \leq a \leq r_{Q}$. Then $E_{i}=\left\langle e_{r_{i-1}+1}, \ldots, e_{r_{i}}\right\rangle$ and $Q_{a}=\left\langle q_{s_{a-1}+1}, \ldots, q_{s_{a}}\right\rangle$, where $q_{p}:=e_{m+p}$ for $p=1, \ldots, n-m$.

Let $\Pi$ be the index set of $(i, a)$ such that $\mathfrak{n}_{\mathbf{a}}=\oplus_{(i, a) \in \Pi}\left(E_{i}^{*} \otimes Q_{a}\right)$. Then $\mathfrak{m} / \mathfrak{n}_{\mathbf{a}}=\oplus_{(i, a) \notin \Pi}\left(E_{i}^{*} \otimes Q_{a}\right)$. As a subspace of $\mathfrak{n}_{\mathbf{a}}^{*} \otimes \mathfrak{m} / \mathfrak{n}_{\mathbf{a}}, \mathfrak{m}_{\mathbf{a}}=$ $\left(\oplus_{i<j} E_{i}^{*} \otimes E_{j}\right) \oplus\left(\oplus_{b<a} Q_{b}^{*} \otimes Q_{a}\right)$ is equal to

$$
\left(\oplus_{i<j} E_{i}^{*} \otimes E_{j} \otimes\left\langle I d_{\oplus_{a} \in \Pi_{i, j}} Q_{a}\right\rangle_{\mathbb{C}}\right) \oplus\left(\oplus_{b<a}\left\langle I d_{\oplus_{i \in \Pi_{b, a}} E_{i}}\right\rangle_{\mathbb{C}} \otimes Q_{b}^{*} \otimes Q_{a}\right)
$$

where $\Pi_{i, j}=\{a:(i, a) \notin \Pi,(j, a) \in \Pi\}$ and $\Pi_{b, a}=\{i:(i, b) \in$ $\Pi,(i, a) \notin \Pi\}$.

We may choose the order of the set of roots of $S L(n)$ in such a way that the maximal root is located in the most left and the lowest box $E_{1}^{*} \otimes Q_{s}$ and the minimal root is located in the most right and the highest box $E_{r}^{*} \otimes Q_{1}$. Then the highest weight vector in $\mathfrak{m}_{\mathbf{a}} \subset \mathfrak{n}_{\mathbf{a}}^{*} \otimes \mathfrak{m} / \mathfrak{n}_{\mathbf{a}}$ is either

$\sum_{a \in \Pi_{i, j}} \sum_{q_{p} \in Q_{a}} x_{\alpha_{p}}^{*} \otimes x_{\beta_{p}}$, where $x_{\alpha_{p}}=e_{r_{j}}^{*} \otimes q_{p}$ and $x_{\beta_{p}}=e_{r_{i-1}+1}^{*} \otimes$ $q_{p}$ for some $i<j$, or,

$\sum_{i \in \Pi_{b, a}} \sum_{e_{p} \in E_{i}} x_{\alpha_{p}}^{*} \otimes x_{\beta_{p}}$, where $x_{\alpha_{p}}=e_{p}^{*} \otimes q_{s_{b-1}+1}$ and $x_{\beta_{p}}=$ $e_{p}^{*} \otimes q_{s_{a}}$ for some $b<a$,

For example, consider $\mathbf{a}=\left(9^{2}, 7^{2}, 3^{4}\right)$ in $P(10,19)$. Then, we have $E=\oplus_{i=1}^{4} E_{i}, Q=\oplus_{a=1}^{3} Q_{a}$ and

$$
\Pi=\{(2,1),(3,1),(3,2),(4,1),(4,2),(4,3)\} .
$$

The highest weight vector of $\mathfrak{m}_{\mathbf{a}}$ is like
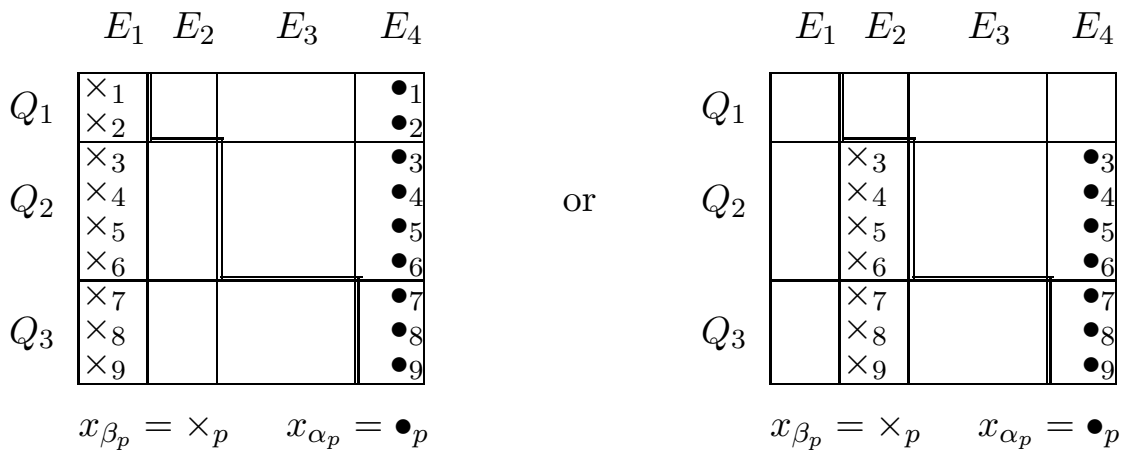

Proposition 4.2. Let $E=\oplus_{i} E_{i}$ and $Q=\oplus_{a} Q_{a}$ be the decomposition associate to $\mathbf{a}$ as in the above and $\left\{e_{1}, \ldots, e_{n}\right\}$ be a basis for $\mathbb{C}^{n}$ indexed as in the above. Then, the highest weight vector of an irreducible $\mathfrak{l}_{\mathbf{a}}{ }^{-}$ representation space in the complement of $\mathfrak{m}_{\mathbf{a}}$ in $\mathfrak{n}_{\mathbf{a}}^{*} \otimes \mathfrak{m} / \mathfrak{n}_{\mathbf{a}}$ is either

(1) a decomposable vector $x_{\alpha}^{*} \otimes x_{\beta}$, where $x_{\alpha}=e_{r_{j}}^{*} \otimes q_{s_{b-1}+1}$ is the lowest weight vector of $E_{j}^{*} \otimes Q_{b}$ and $x_{\beta}=e_{r_{i-1}+1}^{*} \otimes q_{s_{a}}$ is the 
highest weight vector of $E_{i}^{*} \otimes Q_{a}$ for some $(j, b) \in \Pi,(i, a) \notin \Pi$, or,

(2) $\sum_{q_{p} \in Q_{a}} x_{\alpha_{p}}^{*} \otimes x_{\beta_{p}}$, where $x_{\alpha_{p}}=e_{r_{j}}^{*} \otimes q_{p}$ and $x_{\beta_{p}}=e_{r_{i-1}+1}^{*} \otimes q_{p}$ for some $(j, a) \in \Pi,(i, a) \notin \Pi$ such that $(j, a-1) \in \Pi$ and $(i, a-1) \notin$ $\Pi$, or

(3) $\sum_{e_{p} \in E_{i}} x_{\alpha_{p}}^{*} \otimes x_{\beta_{p}}$, where $x_{\alpha_{p}}=e_{p}^{*} \otimes q_{s_{b-1}+1}$ and $x_{\beta_{p}}=e_{p}^{*} \otimes q_{s_{a}}$ for some $(i, b) \in \Pi,(i, a) \notin \Pi$ such that $(i+1, b) \in \Pi$ and $(i+1, a) \notin \Pi$.

Proof. If $i \neq j$ and $a \neq b$, then $\left(E_{j}^{*} \otimes Q_{b}\right)^{*} \otimes\left(E_{i}^{*} \otimes Q_{a}\right)$ is an irreducible $\mathfrak{l}_{\mathbf{a}}$-representation space. The highest weight vector of the irreducible representation spaces $\left(E_{j}^{*} \otimes Q_{b}\right)^{*} \otimes\left(E_{i}^{*} \otimes Q_{a}\right)$ is of type (1).

But if $i \neq j$ and $a=b$, then $\left(E_{j}^{*} \otimes Q_{a}\right)^{*} \otimes\left(E_{i}^{*} \otimes Q_{a}\right) \simeq E_{j} \otimes E_{i}^{*} \otimes\left(Q_{a}^{*} \otimes\right.$ $\left.Q_{a}\right)$ is decomposed as $\left(E_{j} \otimes E_{i}^{*} \otimes\left\langle I d_{Q_{a}}\right\rangle_{\mathbb{C}}\right) \oplus\left(E_{j} \otimes E_{i}^{*} \otimes \operatorname{sl}\left(Q_{a}\right)\right)$, each of which are irreducible $s l\left(E_{j}\right) \times \operatorname{sl}\left(E_{i}\right) \times \operatorname{sl}\left(Q_{a}\right)$-representation spaces. The highest weight vector of the irreducible representation spaces $\left(E_{j} \otimes\right.$ $\left.E_{i}^{*} \otimes \operatorname{sl}\left(Q_{a}\right)\right)$ is of type (1).

The component $E_{i}^{*} \otimes E_{j}$ of $\mathfrak{m}_{\mathbf{a}}$ corresponds to the component $E_{i}^{*} \otimes$ $E_{j} \otimes\left\langle I d_{\oplus_{a \in \Pi_{i, j}} Q_{a}}\right\rangle_{\mathbb{C}}$ in $\mathfrak{n}_{\mathbf{a}}^{*} \otimes \mathfrak{m} / \mathfrak{n}_{\mathbf{a}}$, so its complement in $\oplus_{a \in \Pi_{i, j}} E_{i}^{*} \otimes$ $E_{j} \otimes\left\langle I d_{Q_{a}}\right\rangle_{\mathbb{C}}$ is $\oplus_{a \in \tilde{\Pi}_{i, j}} E_{i}^{*} \otimes E_{j} \otimes\left\langle I d_{Q_{a}}\right\rangle_{\mathbb{C}}$, where $\tilde{\Pi}_{i, j}=\{a:(i, a) \notin$ $\Pi,(j, a) \in \Pi,(i, a-1) \notin \Pi,(j, a-1) \in \Pi\}$ is obtained from $\Pi_{i, j}$ by excluding the smallest index in $\Pi_{i, j}$. The highest weight of the irreducible representation space in these components is of type (2).

Considering the case when $i=j$ and $a \neq b$, we obtain the highest weight vectors of type (3).

q.e.d.

For example, the highest weight vector $\sum_{q_{p} \in Q_{a}} x_{\alpha_{p}}^{*} \otimes x_{\beta_{p}}$ of type (2) is
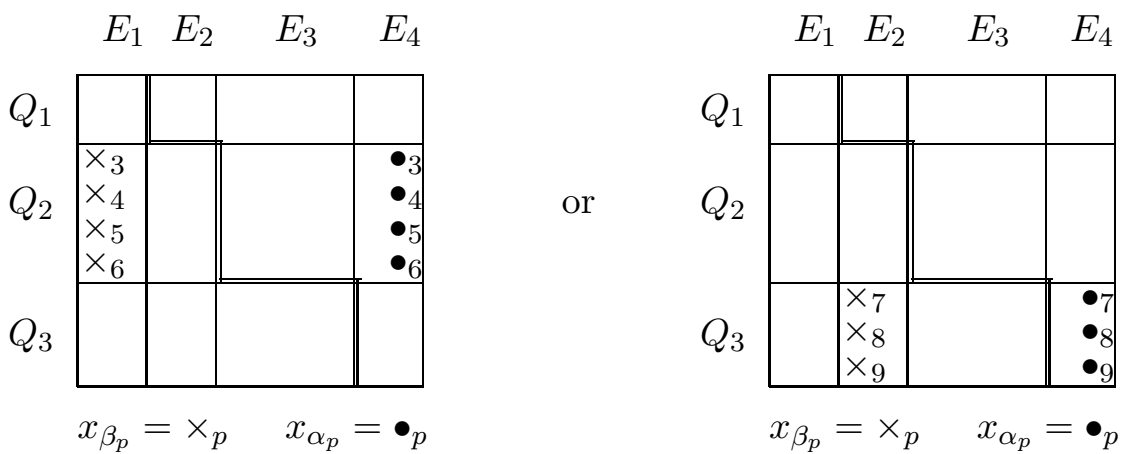

4.2. Proof of the equality $B_{\mathbf{a}}=R_{\mathbf{a}^{*}}$. We will use the same notations as in the previous section. When $\left\{v_{1}, \ldots, v_{k}\right\}$ is a basis of $\mathfrak{n}_{\mathbf{a}}$ such that $v_{1}=x_{\alpha}$, we will use the notation $x_{\beta} \wedge \hat{x}_{\alpha} \wedge \cdots \wedge v_{k}$ to denote the $k$-vector obtained from $v_{1} \wedge \cdots \wedge v_{k}$, which may be considered as a base $k$-vector, by replacing $v_{1}=x_{\alpha}$ with $x_{\beta}$. 
We call the union of all the columns corresponding to $e_{p}$ 's in $E_{i}$ the $E_{i}$-column. Similarly, we call the union of all the rows corresponding $q_{p}$ 's in $Q_{a}$ the $Q_{a}$-row.

Proposition 4.3. Let $\mathbf{a}=\left(p_{1}^{q_{1}}, \ldots, p_{r}^{q_{r}}\right)$ be a partition and let $\mathbf{a}^{\prime}=$ $\left(p_{1}^{\prime} q_{1}^{\prime}, \ldots, p_{r}^{\prime} q_{r}^{\prime}\right)$ be its conjugate. Take a decomposition $E=\oplus_{i} E_{i}$ and $Q=\oplus_{a} Q_{a}$ associated to $\mathbf{a}$ as in the previous section. Let $\Pi$ be the index set of $(i, a)$ such that $\mathfrak{n}_{\mathbf{a}}=\oplus_{(i, a) \in \Pi} E_{i}^{*} \otimes Q_{a}$.

Suppose that for any $(i, a) \notin \Pi$ with $(i+1, a) \in \Pi$ and $(i, a-1) \in \Pi$, both $E_{i}$ and $Q_{a}$ are not one dimensional. Then the Schubert differential system $\mathcal{B}_{\mathbf{a}^{*}}$ is equal to the Schur differential system $\mathcal{R}_{\mathbf{a}}$.

Proof. We will divide the proof into two parts: I. when the highest weight of the irreducible component in the complement of $\mathfrak{m}_{\mathbf{a}}$ in $\mathfrak{n}_{\mathbf{a}}^{*} \otimes$ $\mathfrak{m} / \mathfrak{n}_{\mathbf{a}}$ is of type (1) and II. when it is of type (2) or (3). (See Proposition 4.2 for the types of the highest weight vectors.)

I. Type (1): Fix $(j, b), \in \Pi,(i, a) \notin \Pi$. Let $\left\{v_{1}, \ldots, \ldots, v_{k}\right\}$ be a basis of $\mathfrak{n}_{\mathbf{a}}$ with $v_{1}=x_{\alpha}$ is a lowest weight vector of $E_{j}^{*} \otimes Q_{b}$ and $x_{\beta}$ is the highest weight vector of $E_{i}^{*} \otimes Q_{a}$. We will show that $x_{\beta} \wedge \hat{x}_{\alpha} \wedge \cdots \wedge v_{k}$ has a non-zero component in $\mathbf{I}_{\mathbf{b}}$ for a partition $\mathbf{b} \neq \mathbf{a}$ with $|\mathbf{b}|=|\mathbf{a}|$. Then by Proposition 4.1, $B_{\mathbf{a}^{*}}$ is equal to $R_{\mathbf{a}}$.

$\mathfrak{n}_{\mathbf{a}}$ $\stackrel{\operatorname{ad}\left(x_{\gamma_{4}}\right)}{\longrightarrow}$
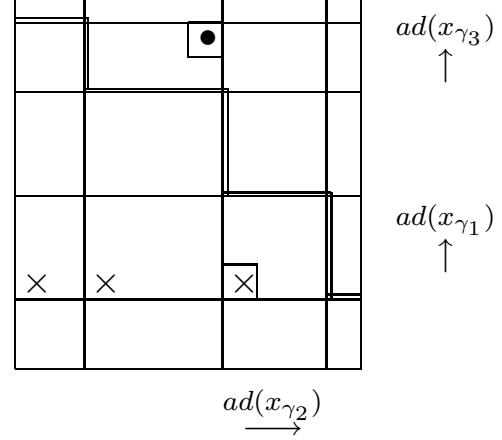

$$
x_{\alpha}=\text { one of } \bullet \text { 's }
$$$$
x_{\beta}=\text { one of } \times \text { 's }
$$

$\mathfrak{n}_{\mathrm{b}}$

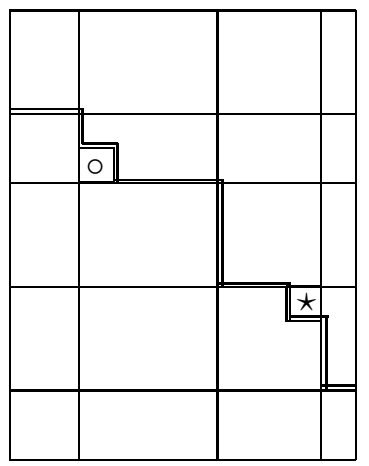

$x_{\beta^{\prime}}=0$

$x_{\alpha^{\prime}}=\star$

Case 1. Assume that $q_{i}^{\prime} \geq 2$ for all $i$. Denote by $x_{\beta^{\prime}}$ the highest weight in the boxes $E_{j}^{*} \otimes Q_{c}$ for all $c$ with $(j, c) \in \Pi$ and by $x_{\alpha^{\prime}}$ the lowest weight vector in the boxes $E_{k}^{*} \otimes Q_{a}$ for all $k$ with $(k, a) \notin \Pi$. Then, there is a partition $\mathbf{b}$ such that $\mathfrak{n}_{\mathbf{b}}=\mathfrak{n}_{\mathbf{a}}-\left\{x_{\beta^{\prime}}\right\} \cup\left\{x_{\alpha^{\prime}}\right\}$. 
By construction, there is $x_{\gamma_{j}}, j=1, \ldots, 4$ in $\mathfrak{p}_{0}$ such that

$$
\operatorname{ad}\left(x_{\gamma_{2}}\right) \operatorname{ad}\left(x_{\gamma_{1}}\right) x_{\beta}=x_{\alpha^{\prime}} \quad \text { and } \quad \operatorname{ad}\left(x_{\gamma_{4}}\right) \operatorname{ad}\left(x_{\gamma_{3}}\right) x_{\beta^{\prime}}=x_{\alpha} .
$$

If all $x_{\alpha}, x_{\beta}, x_{\beta^{\prime}}$ and $x_{\alpha^{\prime}}$ lie neither in the same $E_{i}$-column nor in the same $Q_{a}$-raw as in the picture, then all $\gamma_{i}$ are distinct. Thus,

$\operatorname{ad}\left(x_{\gamma_{4}}\right) \cdots \operatorname{ad}\left(x_{\gamma_{1}}\right)\left(x_{\beta} \wedge \cdots \wedge \hat{x}_{\alpha} \wedge \cdots \wedge v_{k}\right)=x_{\alpha^{\prime}} \wedge \cdots \wedge \hat{x}_{\beta^{\prime}} \wedge \cdots \wedge v_{k}$.

But $x_{\alpha^{\prime}} \wedge \cdots \wedge \hat{x}_{\beta^{\prime}} \wedge \cdots \wedge v_{k}$ is the lowest weight vector of $\mathbf{I}_{\mathbf{b}}$. Since $\operatorname{ad}\left(\mathfrak{p}_{0}\right)$ preserves $\mathbf{I}_{\mathbf{b}}$, there is a non-zero $\mathbf{I}_{\mathbf{b}}$-component in $x_{\beta} \wedge \cdots \wedge \hat{x}_{\alpha} \wedge \cdots \wedge v_{k}$ and thus, it is not contained in $\mathbf{I}_{\mathbf{a}}$.

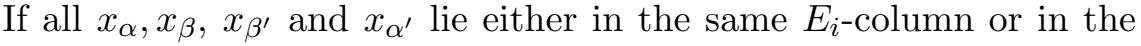
same $Q_{a}$-row, then either $\gamma_{2}$ is equal to $\gamma_{4}$ or $\gamma_{1}$ is equal to $\gamma_{3}$. We consider the case when $\gamma_{2}=\gamma_{4}$ as in the picture (the proof for the other case is similar to this case).

$\mathfrak{n}_{\mathbf{a}}$
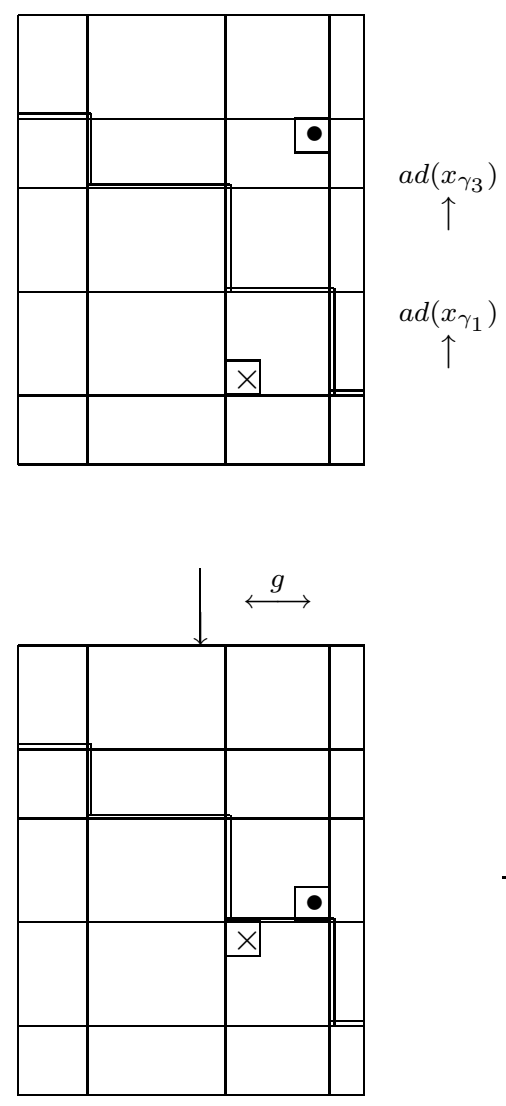

$\mathfrak{n}_{\mathrm{b}}$
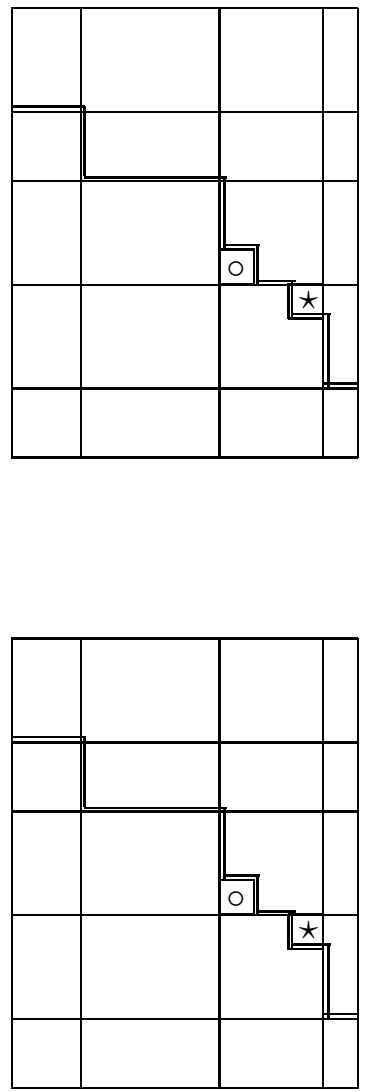

Then the multivector $x_{\alpha^{\prime}} \wedge \hat{x}_{\beta^{\prime}} \wedge \cdots \wedge v_{k} \in \mathbf{I}_{\mathbf{b}}$ can be obtained from the multivector $x_{\beta} \wedge \hat{x}_{\alpha} \wedge \cdots \wedge v_{k}$ by applying $g \circ \operatorname{ad}\left(x_{\gamma_{3}}\right) \operatorname{ad}\left(x_{\gamma_{1}}\right)$, where 
$g \in S L(m) \times S L(n-m)$ which exchanges the most left column $e_{s_{i-1}+1}^{*} \otimes$ $q_{p}, 1 \leq p \leq n-m$ with the most right column $e_{s_{i}}^{*} \otimes q_{p}, 1 \leq p \leq n-m$ in the $E_{i}$-column. This shows that $x_{\beta} \wedge \hat{x}_{\alpha} \wedge \cdots \wedge v_{k}$ is not contained in $\mathbf{I}_{\mathbf{a}}$.

Case 2. If some $q_{a}^{\prime}=1$, then there may be no partition $\mathbf{b}$ with such property as in Case 1. This is the case when $\operatorname{dim} Q_{a}$ is one and $a=b+1$ and $E_{j+1}^{*} \otimes Q_{a} \notin \mathfrak{n}_{\mathbf{a}}$. Then $x_{\alpha^{\prime}}$ is left to $x_{\beta^{\prime}}$ and they are adjacent so we cannot find such a partition $\mathbf{b}$.

$\mathfrak{n}_{\mathbf{a}}$

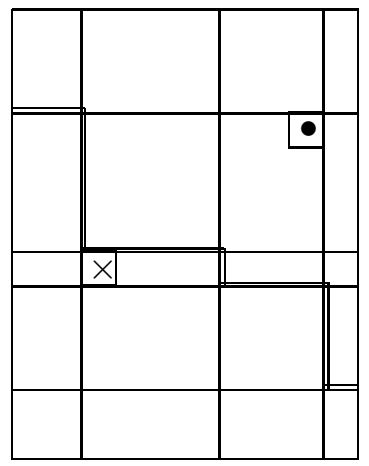

$\mathfrak{n}_{\mathrm{b}}$

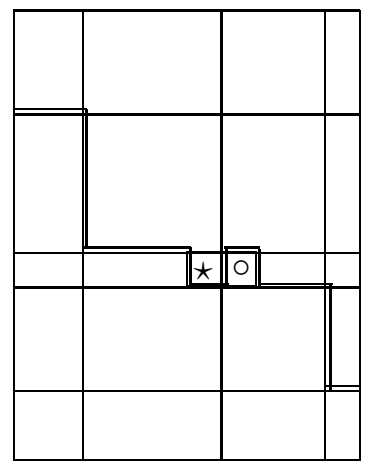

But in this case, consider $\mathbf{b}^{\prime}$ such that $\mathfrak{n}_{\mathbf{b}^{\prime}}=\mathfrak{n}_{\mathbf{a}}-\left\{x_{\beta^{\prime \prime}}\right\} \cup\left\{x_{\alpha^{\prime}}\right\}$ where $x_{\beta^{\prime \prime}}$ is the highest weight vector in the boxes $E_{k}^{*} \otimes Q_{b}$ for all $k$ with $(k, b) \in \Pi$ and $x_{\alpha^{\prime}}$ is the lowest weight vector in the boxes $E_{i}^{*} \otimes Q_{c}$ for all $c$ with $(i, c) \notin \Pi$.

$\mathfrak{n}_{\mathbf{b}^{\prime}}$

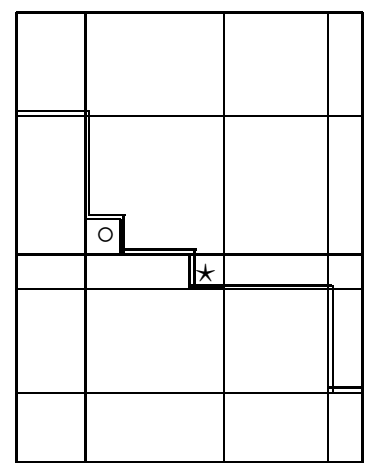

If $i \neq j+1$, then there is such a partition $\mathbf{b}^{\prime}$. If $i=j+1$, then, by the assumption, $\operatorname{dim} E_{i}$ is not equal to one and thus, we can find such a 
partition $\mathbf{b}^{\prime}$. Then by the same argument as in the case 1 , we can prove that $x_{\beta} \wedge \hat{x}_{\alpha} \wedge \cdots \wedge v_{k}$ is not contained in $\mathbf{I}_{\mathbf{a}}$.

II. Type (2) or (3): Let $\sum_{q_{p} \in Q_{a}} x_{\alpha_{p}}^{*} \otimes x_{\beta_{p}}$ be the highest weight vector of type (2) of an irreducible representation space in the complement of $\mathfrak{m}_{\mathbf{a}}$ in $\mathfrak{n}_{\mathbf{a}}^{*} \otimes \mathfrak{m} / \mathfrak{n}_{\mathbf{a}}$, where $x_{\alpha_{p}}=e_{r_{j}}^{*} \otimes q_{p}$ and $x_{\beta_{p}}=e_{r_{i-1}+1}^{*} \otimes q_{p}$ for some $(j, a) \in \Pi,(i, a) \notin \Pi \operatorname{such}$ that $(j, a-1) \in \Pi$ and $(i, a-1) \notin \Pi$. We will show that $\sum_{q_{p} \in Q_{a}} x_{\beta_{p}} \wedge \hat{x}_{\alpha_{p}} \wedge \cdots \wedge v_{k}$ has a non-zero component in $\mathbf{I}_{\mathbf{b}}+\mathbf{I}_{\mathbf{c}}$ if $\operatorname{dim} Q_{a} \geq 2$, and has a non-zero component in $\mathbf{I}_{\mathbf{b}}$, otherwise, for some partition $\mathbf{b}$ and $\mathbf{c}$.

Applying the adjoint actions successively to

$$
\begin{aligned}
\sum_{q_{p} \in Q_{a}} x_{\beta_{p}} \wedge \hat{x}_{\alpha_{p}} \wedge \cdots \wedge v_{k}= & x_{\beta_{s_{a-1}+1}} \wedge \hat{x}_{\alpha_{s_{a-1}+1}} \wedge \cdots \wedge v_{k} \\
& +\sum_{p=s_{a-1}+2}^{p=s_{a}} x_{\beta_{p}} \wedge \hat{x}_{\alpha_{p}} \wedge \cdots \wedge v_{k}
\end{aligned}
$$

we can get

$$
x_{\beta_{s_{a-1}+1}} \wedge \hat{x}_{\alpha_{s_{c}}} \wedge \cdots \wedge v_{k}+x_{\beta_{s_{a-2}+1}} \wedge \hat{x}_{\alpha_{s_{c}}} \wedge \cdots \wedge v_{k}
$$

where $x_{\beta_{s_{a-2}+1}}=e_{r_{i-1}+1}^{*} \otimes q_{s_{a-2}+1}$ and $x_{\alpha_{s_{c}}}=e_{r_{j}}^{*} \otimes q_{\alpha_{s_{c}}}$ and $c$ is the largest index $c$ such that $(j, c) \in \Pi$.

$$
\Uparrow \uparrow
$$
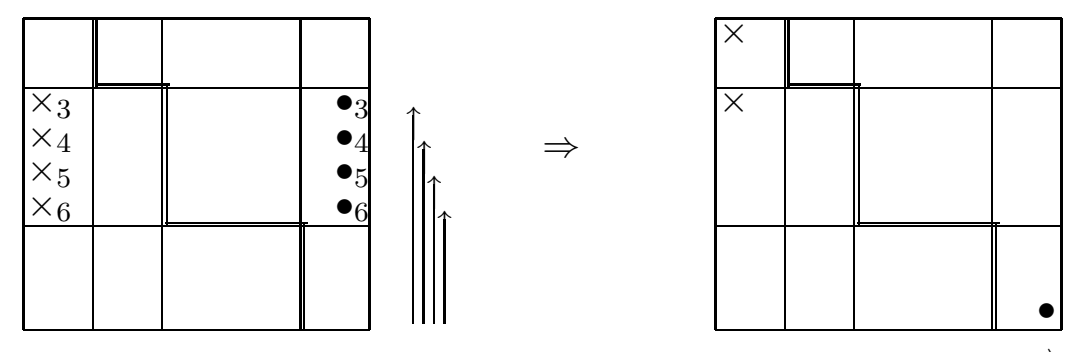

$$
x_{\beta_{p}}=\times_{p} \quad x_{\alpha_{p}}=\bullet p
$$

Applying the adjoint actions again, we can get

$$
y_{\beta_{s_{a-1}+1}} \wedge \hat{y}_{\alpha_{s_{c}}} \wedge \cdots \wedge v_{k}+y_{\beta_{s_{a-2}+1}} \wedge \hat{y}_{\alpha_{s_{c}}} \wedge \cdots \wedge v_{k}
$$

where $y_{\beta_{s_{a-1}+1}}=e_{r_{h}}^{*} \otimes q_{s_{a-1}+1}$ and $y_{\beta_{s_{a-2}+1}}=e_{r_{i}}^{*} \otimes q_{s_{a-2}+1}$ and $y_{\alpha_{s_{c}}}=$ $e_{r_{j-1}+1}^{*} \otimes q_{\alpha_{s_{c}}}$ and $h$ is the largest index $h$ such that $(h, a) \notin \Pi$. This is the sum of the lowest weight vector of $\mathbf{I}_{\mathbf{b}}$ and that of $\mathbf{I}_{\mathbf{c}}$ for some partition $\mathbf{b}$ and $\mathbf{c}$ such that all $\mathbf{a}, \mathbf{b}, \mathbf{c}$ are distinct. 
$\mathfrak{n}_{\mathbf{b}}$

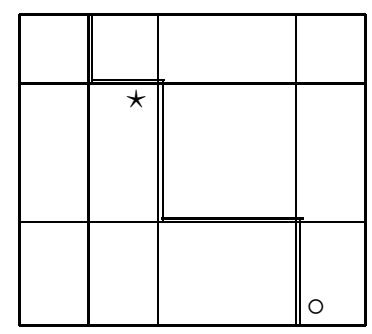

$\mathfrak{n}_{\mathbf{c}}$

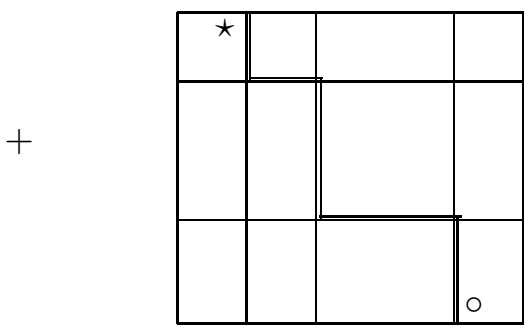

q.e.d.

By Theorem 3.8 and Proposition 4.3, we get

Theorem 4.4. Let $\mathbf{a}=\left(p_{1}^{q_{1}}, \ldots, p_{r}^{q_{r}}\right), p_{r} \neq 0$ be a partition and let $\mathbf{a}^{\prime}=\left(p_{1}^{\prime} q_{1}^{\prime}, \ldots, p_{r}^{\prime} q_{r}^{\prime}\right), p_{r}^{\prime} \neq 0$ be its conjugate. Then $\sigma_{\mathbf{a}}$ is Schur rigid if $q_{i}, q_{i}^{\prime} \geq 2$ for all $i \leq r$.

Remark. One of the problems in algebraic geometry is the smoothability of a singular Schubert variety $X_{w}$ of $G / P$. We say $X_{w}$ is smoothable if there is a smooth subvariety $X$ of $G / P$ with $[X]=\left[X_{w}\right]$ in $H_{*}(G / P, \mathbb{Z})([\mathbf{B}])$. Assume that $\mathbf{a}=\left(p^{q}\right)$ and that $p=1$ or $q=1$, but both are not 1 . Then $\sigma_{\mathbf{a}}$ is a singular Schubert variety and the nonsmoothability of $X_{\mathbf{a}}$ is proved in [B]: if $X$ is a subvariety of $\operatorname{Gr}(m, n)$ with $[X]=\left[\sigma_{\mathbf{a}}\right]$, then $X$ is a Schubert variety of type a. By Theorem 4.4, for a partition $\mathbf{a}=\left(p_{1}^{q_{1}}, \ldots, p_{r}^{q_{r}}\right)$ with its conjugate $\mathbf{a}^{\prime}=\left(p_{1}^{\prime} q_{1}^{\prime}, \ldots, p_{r}^{\prime} q_{r}^{\prime}\right)$, if $q_{i}, q_{i}^{\prime} \geq 2$, for all $i$, then the singular Schubert varieties $\sigma_{\mathbf{a}}$ of type a is not smoothable, neither.

Remark. With the same notations as in Proposition 4.3, if both $E_{i}$ and $Q_{a}$ are one dimensional for some $(i, a) \notin \Pi$ with $E_{i}^{*} \otimes Q_{a}$ adjacent to $\mathfrak{n}_{\mathbf{a}}$, then $B_{\mathbf{a}}$ is a proper subvariety of $R_{\mathbf{a}^{*}}$.

Consider the highest weight vector $x_{\alpha}^{*} \otimes x_{\beta}$ of an irreducible component in the complement of $\mathfrak{m}_{\mathbf{a}}$ in $\mathfrak{n}_{\mathbf{a}}^{*} \otimes \mathfrak{m} / \mathfrak{n}_{\mathbf{a}}$ such that $x_{\alpha} \in \mathfrak{n}_{\mathbf{a}}$ and $x_{\beta} \in E_{i}^{*} \otimes Q_{a} \subset \mathfrak{m} / \mathfrak{n}_{\mathbf{a}}$. Then one can check that $x_{\beta} \wedge \hat{x}_{\alpha} \wedge \cdots \wedge v_{k}$ is contained in $\mathbf{I}_{\mathbf{a}}$. Thus, this gives a non-trivial element in $T_{\mathbf{a}}:=$ $T_{\left[\mathfrak{n}_{\mathbf{a}}\right]} G r(k, \mathfrak{m}) \cap T_{\wedge^{k} \mathfrak{n}_{\mathbf{a}}} \mathbb{P}\left(\mathbf{I}_{\mathbf{a}}\right) \subset \mathfrak{n}_{\mathbf{a}}^{*} \otimes \mathfrak{m} / \mathfrak{n}_{\mathbf{a}}$. Note that as an element of $T_{\left[\wedge^{k} \mathfrak{n}_{\mathbf{a}}\right]} \mathbb{P}\left(\mathbf{I}_{\mathbf{a}}\right)$, this tangent vector gives the map

$$
x_{\alpha} \wedge v_{2} \wedge \cdots \wedge v_{k} \longmapsto x_{\beta} \wedge v_{2} \wedge \cdots \wedge v_{k} \in \mathbf{I}_{\mathbf{a}} / \wedge^{k} \mathfrak{n}_{\mathbf{a}},
$$

where $\left\{v_{1}=x_{\alpha}, v_{2}, \ldots, v_{k}\right\}$ be a basis of $\mathfrak{n}_{\mathbf{a}}$. Then, $c(t)=\left(x_{\alpha}+t x_{\beta}\right) \wedge$ $v_{2} \wedge \cdots \wedge v_{k}, t \in \mathbb{C}$, is a curve in $R_{\mathbf{a}}=G r(k, \mathfrak{m}) \cap \mathbb{P}\left(\mathbf{I}_{\mathbf{a}}\right)$ whose tangent vector is $x_{\alpha}^{*} \otimes x_{\beta}$. But this tangent vector is not contained in $\mathfrak{m}_{\mathbf{a}}$ and thus, $B_{\mathbf{a}}$ is a proper subvariety of $R_{\mathbf{a}^{*}}$. This is a generalization of the counterexamples considered in $[\mathbf{W}]$ or Example 9 of $[\mathbf{B}]$. 


\section{References}

[B] R. Bryant, Rigidity and quasi-rigidity of extremal cycles in compact Hermitian symmetric spaces, to appear in Annals of mathematics studies, 153, Princeton University Press, 2007.

[FH] W. Fulton \& J. Harris, Representation Theory; A first course, Graduate Texts in Mathematics, 129, Springer-Verlag, 1991, MR 1153249, Zbl 0744.22001.

[G] A.B. Goncharov, Generalized conformal structures on manifolds, Selecta Math. Soviet. 6(4) (1987) 307-340, MR 0925263, Zbl 0632.53038.

[H] J. Harris, Algebraic geometry. A first course, Graduate Texts in Mathematics, 133, Springer-Verlag, 1992, MR 1416564, Zbl 0779.14001.

[Ho] J. Hong, Rigidity of smooth Schubert varieties in Hermitian symmetric spaces, math.DG/0410138, to appear in Trans. Am. Math. Soc..

[W] M. Walters, Geometry and uniqueness of some extreme subvarieties in complex Grassmannians, Ph.D. thesis, University of Michigan, 1997.

Research Institute of Mathematics Seoul National University

SAN 56-1 SinRIM-DONG KWANAK-GU

SEOUL, 151-747

KOREA

E-mail address: jhhong@math.snu.ac.kr 